\title{
Anti-angiogenic Targets: Angiopoietin and Angiopoietin Receptors
}

\author{
Elina A. Kiss and Pipsa Saharinen
}

\section{Contents}

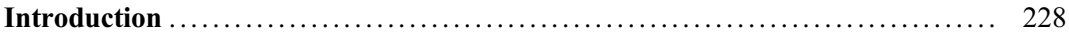

Angiopoietin Growth Factors and TIE RTKs ......................... 229

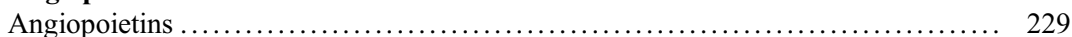

TIE Receptor Signaling ........................................... 229

Phenotypes of Mice with Genetic Deletions of the Angiopoietin-TIE Pathway . . 230

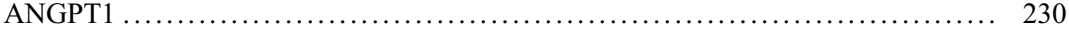

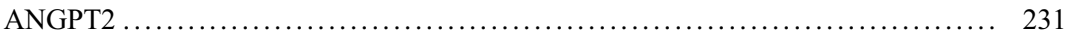

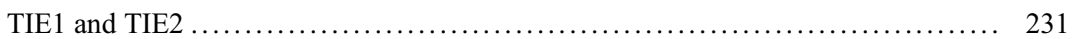

Angiopoietins in Inflammation and Vascular Remodeling ................ 233

Angiopoietins in Experimental Tumor Models ......................... 233

Angiopoietins in Tumor Growth and Angiogenesis .......................... 235

Combined Therapy of ANGPT2 and VEGF or Immune Checkpoint Inhibition . . . . 236

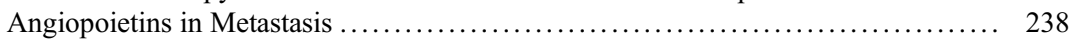

Additional Angiopoietin - TIE Associated Signaling Pathways and Their

Potential as Therapeutic Targets ................................ 239

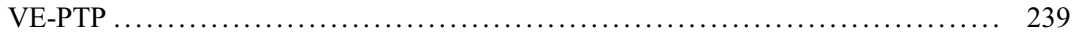

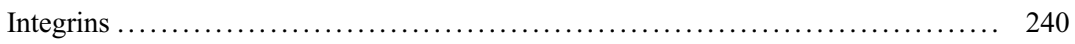

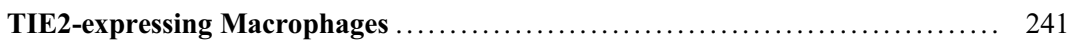

ANGPT2 - an agonist or an antagonist? ........................... 242

\section{E. A. Kiss}

Translational Cancer Biology Program, Research

Programs Unit, Biomedicum Helsinki, University of

Helsinki, Helsinki, Finland

e-mail: elina.kiss@helsinki.fi

\section{P. Saharinen $(\bowtie)$}

Translational Cancer Biology Program, Research

Programs Unit and Medicum Biomedicum Helsinki,

University of Helsinki, Helsinki, Finland

Wihuri Research Institute, Helsinki, Finland

e-mail: pipsa.saharinen@helsinki.fi 


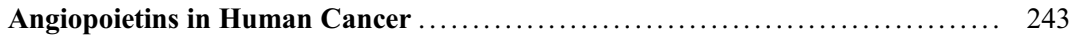

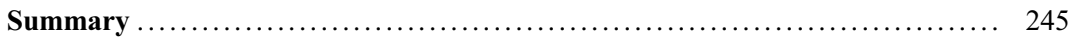

Cross-References ................................................... 245

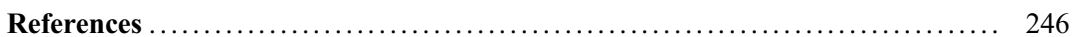

\section{Abstract}

Tumor blood vessel formation (angiogenesis) is essential for tumor growth and metastasis. Two main endothelial ligand-receptor pathways regulating angiogenesis are vascular endothelial growth factor (VEGF) receptor and angiopoietin-TIE receptor pathways. The angiopoietin-TIE pathway is required for the remodeling and maturation of the blood and lymphatic vessels during embryonic development after VEGF and VEGF-C mediated development of the primary vascular plexus. Angiopoietin-1 (ANGPT1) stabilizes the vasculature after angiogenic processes, via tyrosine kinase with immunoglobulin-like and EGF-like domains 2 (TIE2) activation. In contrast, ANGPT2 is upregulated at sites of vascular remodeling. ANGPT2 is secreted by activated endothelial cells in inflammation, promoting vascular destabilization. ANGPT2 has been found to be expressed in many human cancers. Intriguingly, in preclinical models inhibition of ANGPT2 has provided promising results in preventing tumor angiogenesis, tumor growth, and metastasis, making it an attractive candidate to target in tumors. However, until now the first ANGPT2 targeting therapies have been less effective in clinical trials than in experimental models. Additionally, in preclinical models combined therapy against ANGPT2 and VEGF or immune checkpoint inhibitors has been superior to monotherapies, and these pathways are also targeted in early clinical trials. In order to improve current anti-angiogenic therapies and successfully exploit ANGPT2 as a target for cancer treatment, the biology of the angiopoietin-TIE pathway needs to be profoundly clarified.

\section{Keywords}

Angiogenesis · Angiopoietin · Angiopoietin-2 ANGPT · ANG · TIE1 - TIE2 - TEK · Tumor metastasis $\cdot$ Anti-angiogenic therapy

\section{Introduction}

Tumor blood vessels promote tumor growth and, together with tumor associated lymphatic vessels, facilitate metastatic dissemination to distant organs and to lymph nodes. Master regulators of new blood vessel growth (angiogenesis) are vascular endothelial growth factor (VEGF(-A)) and its endothelial cell (EC)-specific tyrosine kinase receptor (RTK), VEGF receptor 2 (VEGFR2) (Ferrara and Adamis 2016). The importance of VEGF in physiological angiogenesis is evident as reduction in the Vegf gene dosage causes aberrations in vascular development and lack of a single Vegf allele in mice results in embryonic lethality (Carmeliet et al. 1996; Ferrara et al. 1996). Hypoxic neoplastic tumor cells as well as tumor-infiltrating inflammatory cells and tumorassociated fibroblasts express VEGF. Excess VEGF stimulates the growth of poorly matured, leaky vessels with irregular blood perfusion, causing hypoxia and stimulating further expression of VEGF in tumors. Due to the vital role of VEGF in tumor angiogenesis, VEGF has been an intensively studied target for tumor anti-angiogenic therapies, and these efforts have led to the approval of VEGF and VEGFR blocking drugs in many human cancers (Ferrara and Adamis 2016). The use of these drugs over several years has led to the understanding that blocking VEGF signaling at most delays disease progression, whereas complete responses are rare. Two types of resistance were postulated to attenuate the 
efficacy of VEGF targeted drugs: evasive resistance, where initial response to anti-angiogenic therapy is lost and disease eventually progresses, and intrinsic resistance, where no antitumor response is observed (Bergers and Hanahan 2008). Several mechanisms of resistance have been suggested based on studies in preclinical models, including the action of other angiogenic factors than VEGF. Angiopoietin growth factors (ANGPT1, ANGPT2 and ANGPT4, originally termed as ANG1, ANG2 and ANG4 (the mouse ortholog was termed Ang3)) and their endothelial receptor TIE2 (also termed TEK) in association with TIE1 represent a second, almost exclusively EC-specific growth factor receptor pathway, which is known to regulate tumor angiogenesis. After angiogenic processes ANGPT1 interacts with TIE2 and promotes vascular stability and endothelial quiescence. ANGPT2 is expressed at low levels in normal tissues but is upregulated in activated ECs during inflammation and in tumor vessels, and indeed elevated ANGPT2 levels are reported in many human cancers. In line with the importance of VEGF-VEGFR and ANG-TIE systems in regulating both physiological and tumor angiogenesis, attempts of blocking both ANGPT2 and VEGF in certain tumor models have been more efficient than blocking either alone. Collectively, combinatorial inhibition of VEGF and ANGPT2 may help to overcome the challenges in current anti-angiogenic therapies, and to improve efficacy of other types of anti-tumor therapies.

\section{Angiopoietin Growth Factors and TIE RTKs}

\section{Angiopoietins}

Angiopoietin growth factors have a vital role in development, maintenance, remodeling, and repair of the blood vessels. Three angiopoietins in human have been described: ANGPT1, ANGPT2, and ANGPT4 (also termed as ANG1, ANG2, and ANG4) (Davis et al. 1996;
Maisonpierre et al. 1997). Additionally in mice Ang3 has been identified, which represents a human ANGPT4 orthologue (Kim et al. 1999). The cellular effects of angiopoietins are mediated by endothelial TIE RTKs. Originally, angiopoietins have been described to bind to TIE2 RTK via the C-terminal fibrinogen-like domain (FLD) of angiopoietins (Kim et al. 2005; Davis et al. 2003). Central coiled-coil (CC) domain, which is connected to the FLD via a linker region, mediates dimerization or trimerization of angiopoietins. Especially ANGPT1 is further clustered to tetramers, pentamers, or higher oligomeric forms via an $\mathrm{N}$-terminal super-clustering domain (SCD) of angiopoietins (Kim et al. 2005). Studies using recombinant angiopoietins have demonstrated the requirement for correct placing of angiopoietin FLDs for TIE2 activation (Davis et al. 2003). Initial experiments showed that a trimeric or tetrameric angiopoietin, with three to four TIE2 binding sites, was effective as a TIE2 agonist in ECs, but more recently a dimeric recombinant agonist form was created (Cho et al. 2004; Oh et al. 2015). ANGPT1 is a strong TIE2 agonist. Although the roles of Ang3 and ANGPT4 are largely unknown, they have been described to act as Tie2 agonists both in vitro and in vivo (Lee et al. 2004). In contrast, ANGPT2 functions as a weak agonist or antagonist, depending on the context (Daly et al. 2006; Yuan et al. 2009). Recently, it has been shown that angiopoietins signal also via integrins. These signaling events have been reported to involve both the FLD and N-terminal angiopoietin domains, but detailed mechanisms of angiopoietin-integrin signaling remain to be further investigated (Felcht et al. 2012; Lee et al. 2014; Hakanpaa et al. 2015).

\section{TIE Receptor Signaling}

Endothelial TIE receptors TIE1 and TIE2 mediate angiopoietin growth factor signaling. They are RTKs with an extracellular domain consisting of two immunoglobulin (Ig)-like domains, three epidermal growth factor domains (EGF), another Ig-like domain, and three fibronectin type III 
(FNIII) domains followed by a transmembrane helix and a split intracellular tyrosine kinase domain (Barton et al. 2006; Macdonald et al. 2006). The ligand binding domain (LBD) includes the three Ig domains and the three EGF domains. These domains fold as a compact, arrowhead-shaped structure that binds the ANGPT1 and ANGPT2 FLDs, utilizing a lockand-key mode of ligand recognition that is unique for RTKs (Barton et al. 2006; Macdonald et al. 2006). All angiopoietins interact with TIE2 but not with TIE1 (Maisonpierre et al. 1997; Davis et al. 1996; Kim et al. 1999; Lee et al. 2004). In fact, no ligand for TIE1 has been so far described. However, TIE1 becomes phosphorylated after ANGPT1 stimulation in primary ECs in a TIE2 dependent manner, but at a lower magnitude than TIE2 phosphorylation (Saharinen et al. 2005; Savant et al. 2015). TIE1 and TIE2 have been shown to interact on the surface of endothelial cells and angiopoietin stimulation increases TIE receptor interaction and receptor translocation to cell-cell contacts (Korhonen et al. 2016; Saharinen et al. 2008). In contacting ECs, ANGPT1 induces the formation of a receptor complex where ANGPT1 activates TIE2 in trans, by bridging TIE2 molecules from opposing endothelial cells in EC-EC junctions (Fukuhara et al. 2008; Saharinen et al. 2008). ANGPT1induced phosphorylation of TIE2 at EC junctions leads to activation of signaling pathways such as PI3K/AKT and eNOS, whereas in motile cells ANGPT1 preferentially activates ECM-bound TIE2 RTKs in cis leading to downstream activation of ERK and DOKR (Master et al. 2001; Babaei et al. 2003; Fukuhara et al. 2008; Saharinen et al. 2008). AKT further phosphorylates the transcription factor Forkhead box protein O1 (FOXO1) involved in metabolic and growth control of endothelial cells (Wilhelm et al. 2016). Phosphorylated FOXO1 is excluded from the nucleus, resulting in suppression of FOXO1 mediated gene transcription, including the FOXO1 targets endothelial cell specific molecule 1 (Esm1) and ANGPT2 (Daly et al. 2004; Korhonen et al. 2016; Kim et al. 2016). Whereas ANGPT1 induces strong TIE2 activation, ANGPT2 is a weak TIE2 agonist. The recently determined crystal structure of the TIE2 FNIII domains has provided evidence for the mechanism of TIE2 activation. The structure revealed that TIE2 dimerization in cis is mediated via the FNIII domains. In the dimeric TIE2 the LBDs were located far apart, facilitating TIE2 activation by multimeric but not dimeric angiopoietins (Leppanen et al. 2017; Moore et al. 2017). In line with previous reports, these results suggest that the lower oligomerization of ANGPT2 explains for its lower agonist activity. However, the TIE2 agonist function of ANGPT2 appears to be important in the lymphatic vasculature during development, whereas in inflammation ANGPT2 inhibits ANGPT1-TIE2 signaling axis via functioning as a TIE2 antagonist (Daly et al. 2006; Thomson et al. 2014; Korhonen et al. 2016; Kim et al. 2016).

\section{Phenotypes of Mice with Genetic Deletions of the Angiopoietin-TIE Pathway}

\section{ANGPT1}

Genetically modified angiopoietin mouse models have revealed a fundamental role for angiopoietins in cardio vascular development. Deficiency of Angpt1 leads to embryonic lethality at E9.5 to E12.5 due to severe cardiovascular defects (Suri et al. 1996). In the absence of ANGPT1, cardiac development is impaired, manifesting as collapsed endocardial-myocardial interactions and a less complex endocardial structure. The mutant mice have also enlarged vessels characterized by poor pericyte coverage, and an immature, low-complexity vascular network. ANGPT1 is expressed in the myocardium of the developing heart, in perivascular cells like pericytes and vascular smooth muscle cells, and in certain other cell types, such as neurons in the retina (Suri et al. 1996; Davis et al. 1996; Kim et al. 2017). In line with the results obtained with the complete ANGPT1 knockout mice, doxycycline-driven ANGPT1 deletion in mice at E10.5 resulted in abnormal vasculature with dilated vessels in various organs and ultimately 
in embryonic lethality between E17.5 and P0. Furthermore, these defects were phenocopied by a cardiomyocyte specific deletion of ANGPT1, suggesting that the vascular defects were induced via hemodynamic changes due to defective heart development in ANGPT1-deficient embryos. Surprisingly, the deletion of ANGPT1 at any point after E13.5 failed to affect the survival of the ANGPT1-deficient mice, suggesting a more specific function for ANGPT1 in the mature, quiescent vasculature (Jeansson et al. 2011). However, ANGPT1 was required for postnatal development of both superficial and deeper vascular networks in the mouse retina (Lee et al. 2013).

\section{ANGPT2}

Mice genetically deficient for $A N G P T 2$ are born at normal frequencies (Gale et al. 2002). However, within the first 2 weeks after birth the ANGPT2deficient mice died due to severe chylous ascites and lymphatic dysfunction associated with patterning abnormalities in collecting lymphatic vessels and lymphatic capillaries. Additionally, the lack of ANGPT2 was observed to impair the postnatal vascular remodeling, namely the regression of the hyaloid vessels in the vitreous of the eye and formation of the retinal vasculature. Backcrossing in the $\mathrm{C} 57 \mathrm{Bl} / 6$ background abolished the severe phenotype of ANGPT2-deficient mice (Fiedler et al. 2006). ANGPT2-deficient C57B1/6 mice develop to adulthood, yet they exhibit impaired response to inflammatory challenges. ANGPT2 was shown to promote the endothelial cell responsiveness to tumor necrosis factor- $\alpha$ (TNF- $\alpha$ ), such as upregulation of EC adhesion molecules. Interestingly, constitutive transgenic expression of ANGPT2 in mouse endothelium resulted in embryonic lethality at E9.5-E10 with major deficiency in vascular formation, a phenotype very similar to that observed in ANGPT1- or TIE2-deficient embryos (Maisonpierre et al. 1997). Besides the vital role of ANGPT2 during vascular and lymphatic development, the studies show that ANGPT2 remains as an important player in the physiology of adult vasculature. Adult mice with inducible endothelial specific expression of human ANGPT2 were reported to have impaired recovery after limb ischemia, namely reduced blood vessel growth and maturation after injury resulting in prolonged perfusion deficiency (Reiss et al. 2007). Another study showed that endothelial overexpression of human ANGPT2 in mice led to leaky vessels with compromised pericyte coverage and hemodynamic problems leading to systemic hypotension (Ziegler et al. 2013).

\section{TIE1 and TIE2}

TIE2-deficient mice die between E10.5 and E12.5 due to the defective development of the cardiovascular system (Dumont et al. 1994). The early vascular development occurs normally, but in the absence of TIE2, the primary capillary plexus fails to mature. The arteriovenous specification is defective resulting in impaired venous development in the TIE2 deficient mouse embryos (Chu et al. 2016). In addition to vascular defects, the TIE2-deficient mouse embryos have impaired cardiac development that resembles defects observed in ANGPT1-deficient embryos. Furthermore, chimeric mice derived from wild type (WT) and TIE receptor null embryonic cells showed that TIE2 was dispensable for fetal hematopoiesis, but in the presence of competing WT cells, the TIE receptor null cells failed to contribute to adult hematopoiesis in bone marrow (Puri and Bernstein 2003). Chimeric mice also demonstrated a requirement for TIE receptors in the microvasculature during late embryonic development and in the blood vessels in adult mice (Puri et al. 1999). In mice lacking TIE1 the initial phases of vascular development occur normally, but the newly formed vessels lose their integrity, which results in lethal hemorrhage between E13.5 and birth (Puri et al. 1995). After embryonic development TIE1 expression decreases but continues to be significantly expressed in some vascular beds, such as in the lungs, and is upregulated at sites of vascular remodeling and by disturbed blood flow in vessel bifurcations (Korhonen et al. 1992; Porat et al. 2004). The loss of endothelial TIE1 in adult mice does not influence the healthy vasculature 
but leads to inhibition of tumor angiogenesis and growth (D'Amico et al. 2014), as well as attenuated atherosclerosis in ApoE-deficient background (Woo et al. 2011).

As well as the lack of ANGPT1 and ANGPT2, the absence of TIE1 impairs the postnatally occurring retinal vascular development in mice (D'Amico et al. 2014). However, the retinal vascular defects were more severe in mice where both TIE1 and TIE2 were deleted (Savant et al. 2015). In the retinal vasculature, ANGPT2 expression is enriched in tip cells (del Toro et al. 2010; Holopainen et al. 2012), whereas TIE2 is low in the leading vascular front. In fact, a distinct role for TIE1 was proposed in the angiogenic front, where it attenuated the expression and cell surface presentation of TIE2, in comparison to remodeling stalk cells, where TIE1 enforced TIE2 signaling (Savant et al. 2015).

Besides the fundamental role of angiopoietinTIE pathway in blood vasculature, it is also needed in lymphatic vessel formation. Reducing the gene dosage of the orphan Tiel during embryonic development results in an early lymphatic phenotype at E12.5, hemorrhagies by E13.5, and lethality (Puri et al. 1995; Qu et al. 2010; D'Amico et al. 2014). In line with these studies, mice where the intracellular part of TIE1 was conditionally deleted or mice where TIE1 was deleted in developing lymphatic valves showed lymphatic defects, including subcutaneous edema and impaired formation of collecting lymph vessels and valve morphogenesis. Additionally, postnatal deletion of TIE1 led to defects in collecting lymphatic vessel maturation (Shen et al. 2014; Qu et al. 2015). More evidence for the importance of the angiopoietin-TIE pathway in lymphatic vasculature was provided by analysis of mice where ANGPT1, or both ANGPT1 and ANGPT2, were simultaneously deleted at E16.5 or where TIE2 was deleted at the time of birth or in adult mice (Thomson et al. 2014; Souma et al. 2016; Thomson et al. 2017; Kim et al. 2017). In these mice, the defective angiopoietin-TIE pathway leads to elevated intraocular pressure as well as impaired ocular drainage, due to the defects of lymphatic capillaries in the corneal limbus or of the Schlemm's canal, a hybrid vessel responsible for the drainage of aqueous humor in the anterior chamber of the eye. Consequently, the mice developed glaucoma. Importantly, loss-of-function mutations in TIE2 or ANGPT1 were found in patients with primary congenital glaucoma (PCG), a worldwide cause of childhood blindness due to impaired function of the Schlemm's canal (Souma et al. 2016; Thomson et al. 2017).

Recent studies have shed light on the role of TIE1 in regulating angiopoietin signaling in postnatal vascular development (D'Amico et al. 2014; Savant et al. 2015) as well as in adult mouse vasculature (Korhonen et al. 2016). Results from TIE1-deficient mice suggest that TIE1 positively contributes to angiopoietin signaling. It was observed that the conditional TIE1 deletion in mouse endothelium reduced TIE2 phosphorylation that was induced by administration of recombinant ANGPT1 in mice, indicating that TIE1 intensifies ANGPT1-induced TIE2 activation (D'Amico et al. 2014; Korhonen et al. 2016). Furthermore, TIE1 was required for ANGPT1 and ANGPT2 induced (delivered via adenoviral vectors in mice) vascular remodeling of tracheal vessels, and for ANGPT2 agonist activity in transgenic mice, where ANGPT2 expression was induced in ECs (Korhonen et al. 2016). Recently, mechanisms of TIE1 signaling have been elucidated. Angiopoietins were found to increase the direct interaction of TIE1 and TIE2 in cell junctions of cultured ECs (Korhonen et al. 2016). Loss of TIE1 impaired TIE2 trafficking in ECs, resulting in decreased TIE2 signaling. The lack of TIE1 led also to compromised TIE2 and AKT phosphorylation upon ANGPT1 binding (Savant et al. 2015; Korhonen et al. 2016). The decreased AKT phosphorylation in TIE1 silenced ECs resulted in activation and nuclear translocation of the AKT target FOXO1, which has been shown to regulate ANGPT2 expression and endothelial cell homeostasis (Korhonen et al. 2016). These results suggest that TIE1 is required for angiopoietin-induced responses in vitro and in vivo, but detailed mechanisms and vascular processes require further studies. 


\section{Angiopoietins in Inflammation and Vascular Remodeling}

Whereas ANGPT1 acts as a paracrine growth factor to stabilize vessels after angiogenic processes, ANGPT2 is secreted in an autocrine fashion in ECs undergoing endothelial activation or vascular remodeling (Fig. 1a, b) (Maisonpierre et al. 1997; Jeansson et al. 2011). In ECs, ANGPT2 is stored in intracellular secretory granules, Weibel-Palade bodies, and the release of ANGPT2 is associated with a type I response of ECs, triggered by inflammatory mediators including histamine (Fiedler et al. 2004). Decreased ANGPT1-TIE2 signaling and reduced ANGPT1/ ANGPT2 ratio, resulting from increased ANGPT2 expression and secretion, is observed in various diseases associated with vascular dysfunction. High ANGPT2 expression has been described in inflammatory disorders, such as sepsis, malaria, acute kidney and lung injuries, acute respiratory distress syndrome (ARDS) as well as in diabetes and in vascular malformations like cerebral cavernous malformations (for references see Saharinen et al. 2017). Furthermore, low levels of TIE1 and TIE2 receptor expression have been linked to increased vascular complications in hemorrhagic Ebola virus infection in mice (Rasmussen et al. 2014). In humans, singlenucleotide polymorphisms associated with low TIE2 expression were associated with risk for ARDS in intensive care unit patients (Ghosh et al. 2016).

Interestingly, mutations in the human TIE2 gene have been identified in both hereditary and sporadic forms of vascular malformations. TIE2 mutations, which result in increased activity of TIE2 or mutations of the downstream target PIK3CA, cause venous malformations, characterized by enlarged venous channels, surrounded by irregularly distributed vascular smooth muscle cells (Castillo et al. 2016; Castel et al. 2016; Limaye et al. 2009). On the contrary, mutations leading to inactivation of TIE2 or ANGPT1 have been associated with PCG in humans (Souma et al. 2016; Thomson et al. 2017). These observations clearly indicate the importance of the delicate balance of angiopoietin-TIE signaling.
In both acute and chronic inflammation a switch from ANGPT1-TIE2 signaling to ANGPT2 antagonist signaling occurs (Fig. 1a, b) (Korhonen et al. 2016; Kim et al. 2016). In LPS-induced acute endotoxemia in mice, membrane bound TIE1 is rapidly cleaved, leading to impaired TIE2 phosphorylation and signaling, whereas Mycoplasma infection of mouse airways results in slower TIE1 cleavage. At the same time, TIE1, TIE2, and ANGPT1 expression is downregulated, which contributes to the loss of TIE2 signaling (Kim et al. 2016; Korhonen et al. 2016). ANGPT2 is readily released from Weibel-Palade bodies after LPS challenge and additionally, the expression of ANGPT2 is increased. ANGPT2, by competing with ANGPT1 in binding to TIE2 decreases TIE2 activation, leading to activation of FOXO1 and upregulation of Angpt2 gene transcription via a positive feedback loop (Daly et al. 2004; Korhonen et al. 2016; Kim et al. 2016). Importantly, inflammation-induced loss of the TIE receptors appears to switch ANGPT2 from an agonist into an antagonist, impairing EC barrier function. In fact, increased ANGPT2/TIE2 ratio has been shown to promote endothelial destabilization via activating endothelial $\beta 1$-integrin (Hakanpaa et al. 2015). Moreover, mice with heterozygous genetic deletion of one Angpt2 allele, in comparison to WT mice, were observed to have advantage of survival in various sepsis models, indicating that ANGPT2 is harmful in inflammation (David et al. 2012). In contrast, TIE2 heterozygous mice lacking one Tie 2 allele were more susceptible to LPS-induced sepsis (Ghosh et al. 2016).

\section{Angiopoietins in Experimental Tumor Models}

Majority of the knowledge of angiogenesis in cancer has been obtained using methods where tumor cells are transplanted into isogenic or immunodeficient mice. In the syngeneic model, the tumor cells and the recipient mice have a common genetic background, which allows the investigation of the effect of the tumor microenvironment on tumor growth. Immunodeficient 


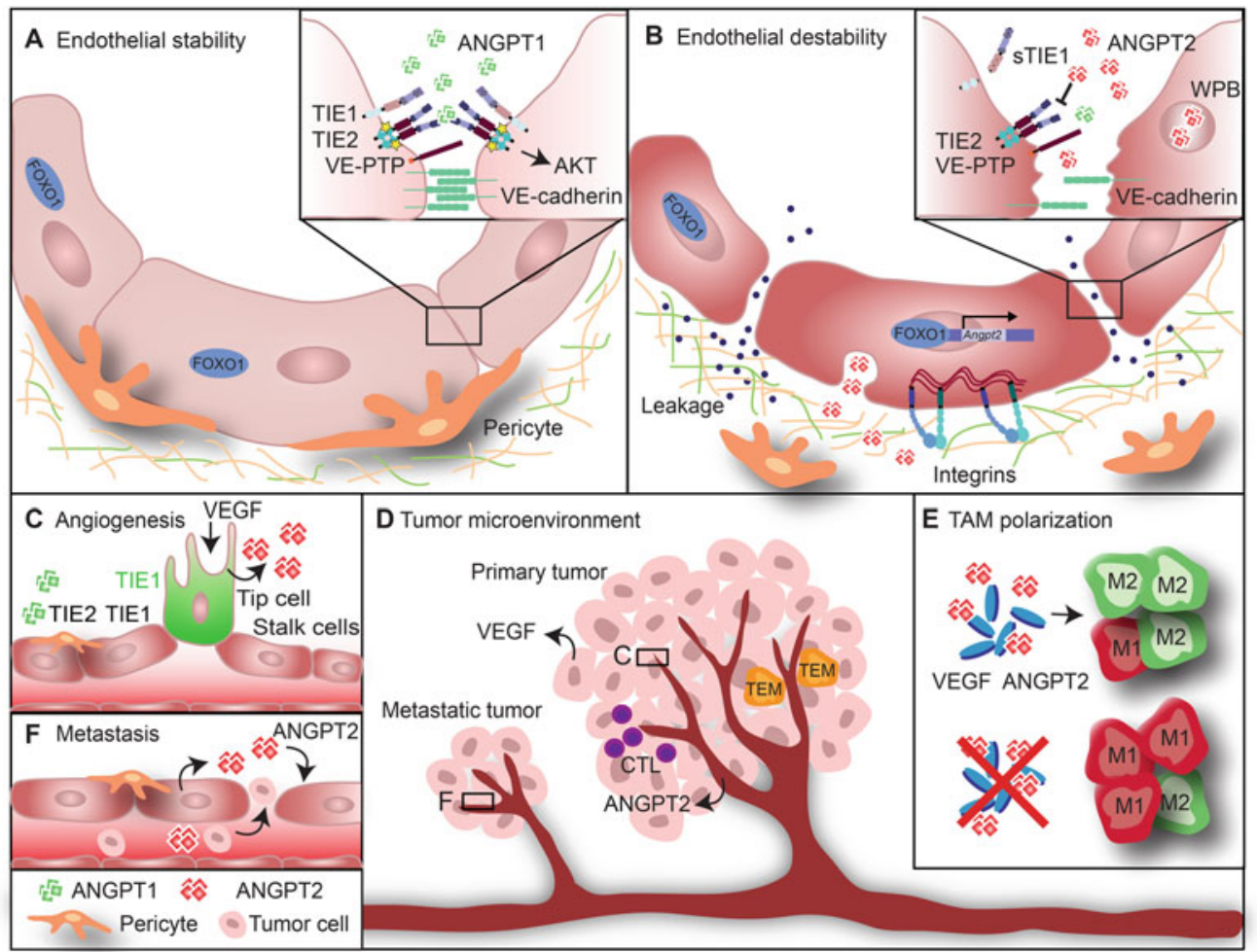

Fig. 1 The role of angiopoietins in angiogenesis and tumor microenvironment. (a) Perivascular cell produced ANGPT1 binds to and activates TIE2 to stabilize newly formed vessels and to limit pathological vascular responses by promoting endothelial barrier function. ANGPT1 binding to TIE2 induces an interaction between TIE2 and the orphan TIE1, enhancing TIE2 activation in endothelial cell-cell junctions. The active TIE2 induces, via the serine kinase AKT, phosphorylation of the transcription factor Forkhead box protein $\mathrm{O} 1$ (FOXO1), which remains in the cytoplasm. VE-PTP serves as a negative regulator of TIE signaling, but promotes the endothelial barrier function via VE-cadherin. (b) In activated endothelium the contextdependent agonist/antagonist ANGPT1 is released from Weibel-Palade bodies (WPB). ANGPT2 binding to TIE2 attenuates the stabilizing ANGPT1-TIE2 signaling axis. In activated endothelium TIE1 is cleaved, releasing soluble TIE1 ectodomain (sTIE1) and the expression of TIE1, TIE2, and ANGPT1 is downregulated. Moreover, in the inflammatory conditions, ANGPT2 switches into a TIE2 antagonist, further impairing TIE2 signaling. In response to diminished TIE2 signaling, FOXO1 translocates to the nucleus and promotes ANGPT2 expression via a positive feedback loop. Decreased ANGPT1-TIE2 signaling impairs endothelial cell-cell junction integrity and the stabilizing cortical actin cytoskeleton (not shown), whereas ANGPT2 signaling via endothelial integrins promotes actin stress fibers, and pericyte dropout. In tumors, blocking ANGPT2 promotes normalization of the tumor vasculature that is mediated via ANGPT1, including decreased leakiness and increased pericyte coverage of the vessels. (c) ANGPT2 is expressed in tip cells of angiogenic sprouts. TIE2 expression in tip cells is downregulated, but it is expressed in the stalk cells. Despite low TIE2 expression, TIE1 is present in sprouting tip cells. Inhibition of ANGPT2 in experimental tumor models decreases sprouting angiogenesis and induces vessel regression. (d) ANGPT2 blocking also interferes with pro-tumorigenic TIE2 expressing macrophages (TEM) that associate with tumor vasculature. In addition, combined blocking of ANGPT2 and VEGF has been found to promote antitumor immunity via increased numbers of tumor-infiltrated activated cytotoxic $\mathrm{T}$ cells (CTL) ANGPT2 inhibition in combination with TIE2 activation (using the antibody ABTAA) has been shown to decrease the number of regulatory T cells in some tumor models. (e) ANGPT2 together with VEGF have been linked to polarization of tumor-associated macrophages (TAM). It has been found that in the presence of ANGPT2 and VEGF, the proportion of protumor M2 macrophages is increased whereas the inhibition of ANGPT2 and VEGF promotes the antitumor M1 phenotype in macrophages. (f) Besides angiogenesis, ANGPT2 promotes lymphangiogenesis, lymph node and distant metastasis. ANGPT2 blocking antibodies or TIE2 activation reduce metastatic spread in preclinical models 
mice are used in xenograft transplantation, where human-derived tumor cells or a piece of solid tumor tissue is used to introduce tumors into mice. Although this system has benefits in using tumors of human origin, the lack of immune cell involvement remains a significant limitation of the system. Despite the weaknesses of the mouse models, they are and remain as an important way to investigate tumor biology. The role of angiopoietins in tumor progression has been dissected using different approaches. Genetically engineered mouse models lacking the expression of ANGPTs or TIE receptors, or mice overexpressing ANGPT2 have provided vital information on the role of angiopoietins in tumor growth, metastasis and tumor angiogenesis. Notably, studies made with angiopoietin inhibiting antibodies or peptibodies developed for therapeutic exploitation have demonstrated the potential benefit of angiopoietin targeting in preclinical models.

\section{Angiopoietins in Tumor Growth and Angiogenesis}

Angiopoietins have a well-established role in regulating tumor growth and angiogenesis (Fig. 1c). Initial direct evidence that host-derived ANGPT2 signaling is needed for tumor growth came from studies with ANGPT2-deficient mice, where tumors grew slower than in WT mice (Nasarre et al. 2009). The growth retardation occurred specifically during early stages of tumor development. In later stages the tumor growth rates were similar in WT and in ANGPT2-deficient mice. The lack of ANGPT2 led to decreased microvessel diameter and more mature vessels with pericyte coverage, suggesting that absence of ANGPT2 leads to normalized phenotype of the tumor vasculature. Another study observed that while overexpression of ANGPT1 in mammary carcinoma cells resulted in stable tumor vasculature, the forced expression of ANGPT2 in tumor cells resulted in aberrant, leaky blood vessels lacking pericytes (Reiss et al. 2009). This report gives further evidence that ANGPT1 signaling promotes vessel stabilization and ANGPT2 the opposite. In line with the data obtained from ANGPT2-deficient mice, the opposite approach of endothelial specific overexpression of ANGPT2 in mice promoted tumor growth (Holopainen et al. 2012).

Several pharmacological approaches to inhibit ANGPT2 function have been investigated in different human tumor xenograft models and in orthotopic syngeneic and transgenic tumor models in mice. The evidence from studies employing ANGPT2-targeting agents indicates a significant role for ANGPT2 in tumor growth and angiogenesis. In general, inhibition of ANGPT2 has been shown to induce reduced microvessel density decreased vascular sprouting, vessel regression, normalization of the remaining tumor vasculature, uniform vessel diameters, reinforcement of the cell-cell junctions, tight association of pericytes with tumor blood vessels, reduction in tumor cell proliferation, and increase in tumor cell apoptosis (Holopainen et al. 2012; Falcon et al. 2009; Hashizume et al. 2010; for additional references see Saharinen et al. 2017). Several ways to block ANGPT2 function have been successfully used. First selective ANGPT2 neutralizing peptide-Fc fusion protein (peptibody) was identified by Oliner et al. and was shown in experimental tumor models to hinder tumor endothelial cell proliferation and restrict tumor growth (Oliner et al. 2004). AMG386, a peptibody, which blocks the binding of both ANGPT1 and ANGPT2 to TIE2 was exploited by pharmaceutical industry to develop trebananib for clinical studies with human patients (Herbst et al. 2009; Coxon et al. 2010). A fully human anti-ANGPT2 monoclonal antibody 3.19 .3 , selectively binding to C-terminal fibrinogen-like domain of ANGPT2, inhibited angiogenesis and induced vessel regression in different orthotopic and transgenic mouse tumor models (Brown et al. 2010; Mazzieri et al. 2011). Antibody administration also significantly hindered spontaneous metastasis (Mazzieri et al. 2011). In a highly metastatic lung carcinoma (LNM35) xenograft model ANGPT2 blocking with a MEDI3617 antibody resulted in reduced tumor growth and increased vessel regression (Holopainen et al. 2012). Additionally, blocking 
ANGPT2 inhibited tumor lymphangiogenesis. Inhibition of ANGPT2 activity with fully human LC06 or LC08 species cross-reactive antibodies resulted in tumor growth inhibition in mouse colorectal and mammary xenograft tumor models (Thomas et al. 2013). The effect of treatment with LC06, specifically targeting ANGPT2, was superior to the treatment with LC08 recognizing both ANGPT1 and ANGPT2. This may at least in part be due to the vessel-stabilizing role of ANGPT1 signaling in the vasculature. In Colo205 colorectal cancer xenograft model, the inhibition of ANGPT2 with a peptibody (L1-7 (N)) resulted in fewer tumor vessels and promoted normalization of the remaining tumor vasculature, characterized by uniform vessel diameters, reinforcement of the cell-cell junctions, reduced sprouting, and tightly associated pericytes (Falcon et al. 2009). While inhibition of ANGPT1 alone had little effect on the tumor vasculature, the combined inhibition of ANGPT1 and ANGPT2 resulted in reduced tumor vasculature (Falcon et al. 2009; Coxon et al. 2010). However, tumor vessel normalization did not occur in the absence of ANGPT1 signaling (Falcon et al. 2009). This suggests, that ANGPT2, but not ANGPT1, regulates the vascularization of the tumors, but the vessel normalization observed in the absence of ANGPT2 is ANGPT1-dependent.

TIE1 deletion has also been shown to affect tumor angiogenesis and growth (D'Amico et al. 2014). The absence of endothelial TIE1 decreased vessel density and vascular perfusion in tumors grown in Tiel gene-targeted mice. Endothelial TIE1 deficiency impaired endothelial cell survival in tumors, and consequently decreased tumor cell survival. Angiogenic sprouting was also reduced in tumors grown in the absence of endothelial TIE1. Targeting of angiopoietins with adenoassociated viral vector delivery of soluble TIE2 receptor (sTIE2) together with the TIE1 deletion resulted in greater tumor growth inhibition than inhibition of either alone. Administration of sTIE2, which by capturing ANGPT1 and ANGPT2 inhibits membrane TIE2 signaling, partially impaired tumor angiogenesis and the growth of primary tumor and tumor metastases in mice (Lin et al. 1998). Interestingly, a recent report shows that an antibody binding to ANGPT2 and simultaneously activating TIE2 (ABTAA) has potent antitumor effects in combination with cytotoxic drugs, by normalizing the tumor vasculature (Park et al. 2017). In an orthotopic glioma model, the combination of temozolomide with ABTAA in comparison to antibody inhibiting ANGPT2 (ABA), reduced the tumor size and vascular leakage while increasing the pericyte coverage of tumor vessels. This tumor vessel normalization further improved perfusion, decreased hypoxia and reduced tumor lactate levels, indicating changes in tumor metabolism. The superior effect of ABTAA to vascular normalization in comparison to $\mathrm{ABA}$ resulted in improved drug delivery into the tumors and survival of mice after glioma cell implantation. Similar effects were also observed in Lewis lung carcinoma model and in the genetic MMTV-PyMT mouse model, where mouse mammary tumor virus (MMTV) LTR is used to drive the expression of the polyoma virus middle T-antigen (PyMT) resulting in the development of mammary tumors. Furthermore, ABTAA stimulated a favorable change in the tumor immune environment, by increasing the proportion of anti-tumorigenic M1 macrophages, and decreasing the numbers of regulatory T cells. ABTAA also decreased, whereas ABA increased, the circulating ANGPT2 levels. These results suggest that in addition to inhibiting ANGPT2, TIE2 activating drugs have beneficial effects on the tumor vasculature and the tumor microenvironment thereby increasing the efficacy of coadministered cytotoxic agents. However, at least in one model, ectopic overexpression of ANGPT1 in mice via an adenoviral vector has been reported to increase metastasis of human lung carcinoma cells (LNM35), due to ANGPT1-induced vessel dilation (Holopainen et al. 2009).

\section{Combined Therapy of ANGPT2 and VEGF or Immune Checkpoint Inhibition}

Results from preclinical tumor models have demonstrated that the dual inhibition of both VEGF and ANGPT2 has additive effects on tumor 
growth inhibition, suggesting that combinatorial targeting of several angiogenic pathways may increase the efficacy of anti-angiogenic therapies. A bispecific antibody neutralizing VEGF and ANGPT2 (ANGPT2-VEGF CrossMab) retarded tumor growth in various tumor models. Especially in larger tumors the combined inhibition of VEGF and ANGPT2 was superior to the respective monotherapies (Kienast et al. 2013). Dual blockade of VEGF and ANGPT2 promoted tumor vessel regression and normalization of vessel architecture. The treatment with ANGPT2 antibody (REGN910) together with aflibercept (VEGF trap consisting of the ectodomain parts of VEGFR1 and VEGFR2) efficiently inhibited the growth of colorectal, prostate, and mammary tumors in xenograft models, decreased tumor vascularity and tumor perfusion (Daly et al. 2013). The combination of these agents was more efficacious than either of the single agent. The authors speculated that ANGPT2-TIE2 signaling increased the survival of tumor endothelial cells, thereby preventing the aflibercept-induced tumor vessel regression. Consequently, compromised EC survival after ANGPT2 blockage might have increased the efficacy of aflibercept treatment. In line with this, another study showed that VEGFR2 inhibition in pancreatic neuroendocrine tumors (PNETs) induced upregulation of ANGPT2 expression that was associated with resistance to VEGFR2 targeted anti-angiogenic therapy (Rigamonti et al. 2014). The combined blockade of ANGPT2 (using the anti-ANGPT2 monoclonal antibody 3.19.3) and VEGFR2 resulted in tumor growth inhibition. Although hypoxia was increased in tumors, the combination treatment did not provoke invasion or metastasis (Rigamonti et al. 2014). Additionally, their analysis of published microarray data suggested that high ANGPT2 expression in nontreated human sarcoma predicted poor response to VEGF neutralization therapy. In colon carcinoma xenograft model dual inhibition of ANGPT2 (using L1-7 (N)) and VEGF (using a monoclonal anti-VEGF antibody) caused tumor growth rate retardation, associated with reduction in cell proliferation and increase in tumor cell apoptosis (Hashizume et al. 2010).
High numbers of tumor associated macrophages (TAMs) have been linked to increased vascular density in human tumors (De Palma et al. 2017), and recent reports propose a role for tumor macrophages in the success of ANGPT2/ VEGF therapy (Fig. 1d, e). In orthotopic syngeneic glioblastoma model combined therapy with AMG386 (ANGPT1/2 neutralizing peptibody) and aflibercept resulted in strong reduction of F4/80 ${ }^{+}$TAMs (Scholz et al. 2016). Additionally, dual inhibition of ANGPT2 and VEGF decreased the number of vascular sprouts and vessels, and reduced vessel permeability via normalization of the tumor vasculature. In another study, mice with either orthotopic syngeneic glioblastoma or human xenograft glioblastoma were treated with antibodies inhibiting either VEGF alone (B20) or bispecific antibodies inhibiting both ANGPT2 and VEGF (A2V) (Kloepper et al. 2016). The dual inhibition increased the survival of mice when compared to monotherapy. As a mechanistic explanation for the better survival, it was suggested that A2V treatment favored the classical antitumor M1 macrophages. Consistent results were obtained again with mouse and human glioblastoma models from studies using ANGPT2 neutralizing antibody (MEDI3617) in combination with cediranib (a small-molecule tyrosine kinase inhibitor of the VEGFRs) (Peterson et al. 2016). Combined blocking of ANGPT2 and VEGF signaling increased the survival of tumor implanted mice when compared to either of the monotherapies. Mice receiving dual therapy exhibited improved vessel normalization. Interestingly, the superior effect of combined therapy over monotherapies was lost, when TAM recruitment to tumors was blocked by using an anticolony-stimulating factor-1 (CSF-1) neutralizing antibody, indicating that the improved survival induced by the dual treatment was mediated by TAMs.

The abnormal tumor vasculature can also impair antitumor immunity, by limiting the extravasation of T cells, including $\mathrm{CD} 8^{+}$cytotoxic $\mathrm{T}$ cells into the tumor bed, thereby promoting a state of immunosuppression (Lanitis et al. 2015). Thus, it has been envisioned that anti-angiogenic therapy may enhance antitumor immunity. This was shown in 
a recent study where ANGPT2 and VEGF blockade (using the bispecific antibody A2V) was combined in both genetic and transplant tumor models (Schmittnaegel et al. 2017). A2V induced an anti-angiogenic response normalizing the remaining blood vessels and facilitated perivascular accumulation of activated, interferon- $\gamma$ (IFN $\gamma$ )-expressing $\mathrm{CD}^{+}$cytotoxic T cells. Notably, in an immunogenic tumor model cytotoxic $\mathrm{T}$ cells were required for $\mathrm{A} 2 \mathrm{~V}$ antitumor effects. The therapy also upregulated the expression of the immune checkpoint ligand, programmed cell death ligand 1 (PD-L1), and blocking PD-1 in combination with $\mathrm{A} 2 \mathrm{~V}$ improved tumor control in certain tumor models, suggesting that immune cells may function as effectors of anti-angiogenic therapy.

Hypoxic tumors are often more resistant to radiation therapy. Interestingly, ANGPT2 expression is reported to increase in cancer patients of head and neck squamous cell carcinoma (HNSCC) after radiation therapy (Sridharan et al. 2016). One study observed that VEGFR2 inhibition created a short time window of vessel normalization before damaging the blood vessels, during which radiation therapy was effective (Winkler et al. 2004). This was characterized by an increase in tumor oxygenation, pericyte coverage of tumor blood vessels, and reduction in vessel diameter. VEGFR2 blockade induced upregulation of ANGPT1, which mediated the vessel normalization through TIE2. These data in part demonstrate the complexity of the tumor environment and how different treatment procedures change it. Treatment efficiency is context-dependent and optimal therapy is often a combination of different procedures.

\section{Angiopoietins in Metastasis}

Besides in tumor growth and angiogenesis, ANGPT2 has been shown to promote tumor metastasis (Fig. 1f). First indication that ANGPT2 plays a crucial role in tumor metastasis derived from studies done in MMTV-PyMT mice, which develop mammary tumors metastasizing in lungs. In these mice, treatment with ANGPT2 blocking antibody (clone 3.19.3) inhibited spontaneous tumor metastasis and additionally, restricted the growth of emerging metastases (Mazzieri et al. 2011). There are several other reports pinpointing the crucial role of ANGPT2 in tumor spreading. In mice overexpressing endothelial ANGPT2, the excess of ANGPT2 promoted B16 melanoma cell homing and early tumor growth in the lungs after their intravenous administration in mice (Holopainen et al. 2012). In these mice, ANGPT2 was shown to decrease endothelial barrier integrity, thereby likely enabling tumor metastasis. Additionally, WT mice treated with ANGPT2 expressing adenoviruses showed increased lymph node and lung metastasis of LNM35 tumor cells (Holopainen et al. 2012). Consistently, blocking ANGPT2 with an antibody (MEDI3617) in WT tumor bearing mice reduced tumor lymphangiogenesis and lymph node and lung metastasis, and improved cell junction integrity of pulmonary capillaries in mice with circulating tumor cells that homed to the lungs. ANGPT2 therefore increases metastasis at least, in part, by decreasing capillary integrity. In contrast, global ANGPT1 deficiency was shown to enhance lung metastasis without affecting primary tumor growth. The results suggested that ANGPT1 was required to inhibit the attachment and extravasation of tumor cells through pulmonary capillaries (Michael et al. 2017). Furthermore, tumor vascular normalization induced by ABTAA (the ANGPT2 inhibiting and TIE2 activating antibody), decreased tumor metastasis in the MMTV-PyMT tumors (Park at el. 2016).

ANGPT2 has been also linked to pericyte loss and subsequent vessel destabilization. Pericyte depletion induced by imatinib therapy, which inhibits platelet-derived growth factor receptor $b$ (PDGFR-b) activity, decreased the growth of well-established tumors, but increased lung metastasis (Keskin et al. 2015). ANGPT2 was found upregulated in the imatinib treated tumors, and combination of imatinib with ANGPT2 blockage decreased both metastasis and tumor growth, suggesting that ANGPT2 enhanced metastasis in the destabilized tumor vasculature. In pancreatic ductal adenocarcinoma ANGPT2 was observed to promote lymphatic metastasis (Schulz et al. 2011). ANGPT2 mRNA expression 
was localized in the ductal tumor cells, but not in normal tissue, and in cultured pancreatic cancer cells ANGPT2 was induced by TGF- $\beta$ stimulation. Ectopic ANGPT2, but not ANGPT1, expression in pancreatic cancer xenografts increased peritumoral lymphatic vessel density and lymphatic metastasis, whereas of soluble TIE2 was able to counteract the harmful effects of ANGPT2.

Interestingly, ANGPT2 expression has been also associated with determining the pre-metastatic niches in tissues. Increased ANGPT2 expression induced by calcineurinNuclear factor of activated T-cells (NFAT) signaling was observed in early metastatic sites in lung endothelium, where it promoted angiogenesis and tumor metastasis (Minami et al. 2013). Calcineurin-NFAT signaling was suggested to be activated through increased VEGF expression induced by the primary tumor. The effect of upregulation of ANGPT2 in lung endothelium could be prevented by overexpressing Down syndrome critical region gene 1 (DSCR1), a negative regulator of calcineurin-NFAT signaling or by administration of soluble TIE2.

Combined inhibition of ANGPT2 and VEGF has been found beneficial in preventing tumor metastasis compared to corresponding monotherapies in preclinical models. In a study where H460M2 tumor cells metastasizing to the lungs were subcutaneously implanted in mice, blockade of ANGPT2 and VEGF using a bispecific antibody (CrossMab) in combination with chemotherapy completely eradicated the tumors. Besides being beneficial for reducing primary tumor size, the tumor-derived DNA in blood from circulating tumor cells was decreased following ANGPT2 and VEGF inhibition, indicating that dual blockade of ANGPT2 and VEGF impairs the early dissemination of tumor cells. Moreover, after primary tumor removal, adjuvant anti-ANGPT2-VEGF therapy decreased metastasis. Inhibition of VEGF and ANGPT2 led to regression and normalization of the tumor vessels and due to ANGPT2 inhibition also to reduced metastasis (Kienast et al. 2013). Others also report that ANGPT2 blockade combined with low-dose metronomic chemotherapy and VEGF blockade significantly reduced metastasis in a preclinical mouse model of postsurgical adjuvant therapy (Srivastava et al. 2014). The combination with ANGPT2 inhibition resulted in decreased numbers of all the macrophages and a subset of pro-tumorigenic $\mathrm{CCR}^{+} \mathrm{TIE}^{-}$metastasisassociated macrophages (MAMs) within the metastatic niche. The recruitment of CCR2 ${ }^{+} \mathrm{TIE}^{-}$ MAMs was dependent on CCL2, and ANGPT2 antibody treatment was observed to reduce the CCL2 expression in lung metastases. Additionally, ANGPT2 stimulation induced adhesion molecule expression by endothelial cells, which may further promote the myeloid cell trafficking to the metastases.

Results from ANGPT2-deficient mice unexpectedly showed that metastatic growth of mouse colon carcinoma cells in the liver and associated tumor angiogenesis were enhanced in the absence of ANGPT2 (Im et al. 2013). Furthermore, it was observed that serum levels of circulating G-CSF and CXCL1 were increased in the ANGPT2 deficient mice, which supported the recruitment of myeloid cells into the liver. In contrast with the observation in liver colonization, but in line with previous reports, the tumor growth in lungs was impaired in the absence of ANGPT2. This study underlines the complexity of the ANGPT2-mediated effects on tumors and in vascular beds of various tissues and the need to further carefully evaluate the context-dependent role of ANGPT2.

\section{Additional Angiopoietin - TIE Associated Signaling Pathways and Their Potential as Therapeutic Targets}

\section{VE-PTP}

An important regulator of the ANGPT-TIE pathway, vascular endothelial protein tyrosine phosphatase (VE-PTP) is an EC-specific receptor tyrosine phosphatase that attenuates TIE2 activation and participates in the regulation of vascular homeostasis. VE-PTP is expressed in both arterial and venous endothelium, and its expression is 
elevated in hypoxia. VE-PTP is essential for the cardiovascular development and consequently, the genetic deletion of VE-PTP results in embryonic lethality between E9.5 and E11 (Baumer et al. 2006; Dominguez et al. 2007). The VEPTP-deficient mouse embryos suffer from remodeling defects of the vasculature, namely, the failure to form branched vascular networks. This defect was most pronounced in the yolk sac, where vessels formed large blood cavities without branches.

VE-PTP associates with TIE2 and regulates its activity via dephosphorylation. Additionally, it also associates with VE-cadherin increasing its adhesive function. Antibodies against VE-PTP, which specifically prevent its binding with TIE2 but not with VE-cadherin, provoked increased TIE2 activation, cell proliferation, and vessel enlargement in an allantois explant culture (Winderlich et al. 2009). Blocking VE-PTP function using a small molecular inhibitor (AKB9778) or anti-VE-PTP antibodies stabilized the vascular barrier function in the mouse lungs challenged with LPS and prevented endothelial permeability induced by VEGF. Interestingly, TIE2 was a prerequisite for the effect of VE-PTP inhibition on vessel integrity, whereas VE-cadherin was dispensable, as inhibition of VE-PTP was able to stabilize the vascular endothelium even in mice with conditional deletion of VE-cadherin (Cdh5) (Frye et al. 2015).

Interestingly, the expression of VE-PTP in adult mouse endothelium was upregulated upon exposure to tumor cells, which may contribute to abnormal blood vessel phenotype via dampening vascular stabilizing TIE2 signaling. Indeed, inhibition of VE-PTP using AKB-9778 resulted in normalization of the vessel phenotype in mouse tumor models (Goel et al. 2013). AKB-9778 increased TIE2 activation, which promoted vascular stability. Inhibition of VE-PTP early in primary tumor development transiently delayed tumor growth by stabilizing tumor vessels, but significantly decreased tumor growth when combined with radio or chemotherapy. Additionally, VE-PTP inhibition was observed to delay metastatic progression after primary tumor excision and prolong the survival of mice.
Besides TIE2 and VE-cadherin, VE-PTP has been reported to regulate the VEGF receptor, VEGFR2. In cells stimulated with VEGF, VE-PTP silencing further increased VEGFR2 phosphorylation (Mellberg et al. 2009). Interestingly, VE-PTP, VEGFR2, and TIE2 were found together in complexes and VE-PTP was shown to regulate the activity of VEGFR2 via TIE2 (Hayashi et al. 2013). VE-PTP-deficient embryoid bodies showed disorganized sprouts and defects in lumen formation and polarization. Consistently, teratomas in mice lacking VE-PTP showed disordered and poorly functioning vasculature and noticeable VEGFR2 activation. The authors concluded that one way how TIE2 promotes vascular stability and quiescence is the inhibition of VEGFR2 receptor activation via VE-PTP. Interestingly, it was also shown that the zebrafish VE-PTP orthologue ptp-rb had an important role in regulating EC polarization and lumen formation.

\section{Integrins}

Integrins are cell surface receptors required for cell attachment and migration on the surrounding extracellular matrix, with well-established roles in tumor angiogenesis. Some integrins are highly upregulated in tumor associated blood vessels and they act together with angiogenic growth factor receptors to regulate their responsiveness to the corresponding growth factor ligands (Ivaska and Heino 2011). Recently, several reports have shown that angiopoietins can bind to certain integrins. In breast cancer cells, which do not express TIE2, ANGPT2 associated with integrin $\alpha 5 \beta 1$ leading to the activation of $\alpha 5 \beta 1$ signaling pathway and cancer metastasis in xenografts in mice (Imanishi et al. 2007). Also, in a TIE2-negative glioma cell line, ANGPT2 was observed to bind to $\alpha 5 \beta 1$-integrin (Lee et al. 2014). While the interaction between angiopoietins and TIE2 occurs via the C-terminal FLD, the integrin interacting domains of angiopoietins are not yet fully clarified. Initially, by using short peptides corresponding to amino acid sequences of FLD of ANGPT2, it 
was observed that certain peptides were inhibiting the binding of integrin-expressing glioma cells to ANGPT2 (Lee et al. 2014). These sequences were located outside of the TIE2 binding region of the FLD, and were required for ANGPT2 interaction with $\alpha 5$-integrin. However, another study suggested that ANGPT2, but not ANGPT1, can activate $\alpha 5 \beta 1$-integrin via the ANGPT2 $\mathrm{N}$-terminal domain, which is distinct from the TIE2-binding FLD (Hakanpaa et al. 2015). In this study, chimeras with either ANGPT2 N-terminal domain fused with ANGPT1 FLD or ANGPT1 N-terminal domain fused with ANGPT2 FLD were used to stimulate the fibronectin binding of $\alpha 5 \beta 1$-integrin. The interaction between angiopoietins and integrins is weaker in comparison to that of TIE2 receptor, indicating that TIE2 serves as the primary receptor for angiopoietins (Felcht et al. 2012). However, recently, growing amount of data describes situations where the cell surface TIE expression is diminished, while ANGPT2 is highly upregulated, potentially facilitating ANGPT2 interaction with other receptors. It remains to be seen if the interaction between angiopoietins and integrins could be targeted for future vascular therapies.

In endothelial monolayers where TIE2 was silenced, autocrine ANGPT2 was found to stimulate the formation of $\beta 1$-integrin positive, elongated and centrally located matrix adhesions, which are distinct from the focal adhesions of quiescent EC monolayers. The altered substrate adhesion of the TIE2 silenced cells resulted in actin stress fiber production and decreased endothelial barrier function (Hakanpaa et al. 2015). Independent reports have shown that Angpt2 mRNA (del Toro et al. 2010) and protein (Holopainen et al. 2012) expressions are upregulated in tip cells, whereas TIE2 was located to the stalk cells instead of the tip cells. This suggests that ANGPT2 acts in a paracrine fashion on stalk cells or via other receptors on tip cells, which express low levels of TIE2. In fact, it was observed that $\beta 1$-integrin was upregulated in tip cells (del Toro et al. 2010). Based on the current data available, it is intriguing to hypothesize that the excess of ANGPT2 in tip cells, in the absence of TIE2, interacts and activates integrin receptors leading to tip cell migration, as reported by Felcht et al. They found that activated sprouting tip cells downregulated TIE2 and upregulated ANGPT2 expression (Felcht et al. 2012). TIE2 low ECs were observed to express av $\beta 5, \alpha 5 \beta 1$, and av $\beta 3$ integrins during angiogenesis. In fact, ANGPT2-induced increase in EC migration was dependent on integrin activation. In addition, $\alpha v \beta 3$-integrin has been found in a complex with TIE2 on the cell surface (Thomas et al. 2010). ANGPT2 was shown to induce clustering of TIE2 and $\alpha v \beta 3$-integrin at the EC junctions resulting in FAK activation, dissociation of the integrinassociated adaptor proteins, and $\alpha v \beta 3$-integrin internalization and lysosomal degradation. Regulation of FAK-activity and integrin trafficking may represent a possible model of how ANGPT2 promotes endothelial destabilization.

Integrins have also been designated a role in fine-tuning the TIE receptor signaling. TIE2 and $\alpha 5 \beta 1$ integrin have been observed to form a complex, which is increased upon $\alpha 5 \beta 1$ activation by fibronectin (Cascone et al. 2005). Interaction of TIE2 and $\alpha 5 \beta 1$ facilitated the activation of TIE2 with low ANGPT1 concentrations. Additionally, blocking the $\alpha 5 \beta 1$ integrin function inhibited the ANGPT1-enhanced angiogenesis. Similarly, $\alpha 5 \beta 1$ integrin has also been shown to promote ANGPT1-induced formation of heteromeric complexes of TIE1 and TIE2, TIE receptor activation, and downstream signaling (Korhonen et al. 2016). In another study, both TIE1 and TIE2 were reported to associate with integrins $\alpha 5 \beta 1$ and $\alpha \mathrm{V} \beta 3$ through their ectodomains (Dalton et al. 2016). Furthermore, ANGPT1-mediated angiogenesis of the retinal vasculature that develops postnatally in mice requires $\alpha \mathrm{v} \beta 5$ integrin expression in retinal astrocytes (Lee et al. 2013).

\section{TIE2-expressing Macrophages}

In addition to endothelial cells, angiopoietin receptor TIE2 is expressed by certain hematopoietic progenitors (Ito et al. 2016) and by a unique subset of circulating monocytes (Mazzieri et al. 2011; 
Coffelt et al. 2010; De Palma et al. 2005). Studies in mouse tumor models have demonstrated that circulating monocytes extravasate into tumors in response to various chemoattractants, and differentiate and mature into TAMs, a process influenced by the colony-stimulating factor 1 (CSF1) (De Palma et al. 2017). TIE2expressing monocytes (TEMs) are recruited to tumors and have been observed in human breast cancer specimens (Kim et al. 2013; De Palma et al. 2005). Interestingly, TEMs constitutively circulate in peripheral blood but are not found in quiescent tissues suggesting that they are specifically attracted by cues from sites of tissue remodeling and angiogenesis. In fact, TEMs were observed to be proangiogenic and associate with newly formed vessels in murine tumors. TEMs were suggested to be recruited to tumors by chemokine CXCL12 via their expression of chemokine receptor CXCR4 (Welford et al. 2011). ANGPT2 interaction with TIE2 on TEMs induced TIE2 phosphorylation and upregulation of the expression of various tumor-promoting factors (Coffelt et al. 2010; Venneri et al. 2007; Murdoch et al. 2007). Blockade of ANGPT2 was shown to inhibit the transcriptional upregulation of TIE2 in TEMs and thereby impairing their association with tumor vasculature and their proangiogenic activity (Mazzieri et al. 2011). Additionally, the knockdown of TIE2 specifically in myeloid cells resulted in reduced tumor angiogenesis and perivascular association of TEMs.

In mammary carcinoma, cancer cell intravasation has been found to occur at microanatomical vascular structures, called Tumor MicroEnvironment of Metastasis (TMEM), composed of a tumor cell, a perivascular TEM, and an endothelial cell. TMEMs have been also identified in human mammary carcinomas, correlating with metastasis. Recently, TIE2 blocking using rebastinib, a TIE2 kinase inhibitor, was found to reduce tumor growth and metastasis in an orthotopic metastatic mouse mammary carcinoma. Rebastinib reduced TEM infiltration, suggesting that the anti-tumor effects of rebastinib could be mediated via inhibition of protumoral TEMs (Harney et al. 2017).

\section{ANGPT2 - an agonist or an antagonist?}

Whether ANGPT2 acts as an agonist or antagonist of TIE2 in the tumor microenvironment remains as an interesting question. As discussed above, structural studies have provided insight into the molecular mechanisms behind the weak agonist activity of dimeric ANGPT2, when compared to higher activity of multimeric ANGPT1 (Leppanen et al. 2017). In general, when ANGPT2 levels are increased, such as in cancer and in various vascular leakage associated diseases, the decreased ANGPT1/ANGPT2 ratio favors ANGPT2-TIE2 signaling. This can be recapitulated using cultured endothelial cells, where excess ANGPT2 can inhibit ANGPT1-induced TIE2 activation (Maisonpierre et al. 1997). However, the outcome of ANGPT2 signaling is context-dependent. It has been suggested that the agonist activity of ANGPT2 would be important for normal homeostasis in stressed ECs that are exposed to low ANGPT1 levels (Daly et al. 2006). In addition, results using genetic mouse models have demonstrated that ANGPT2 functions as an agonist in the lymphatic vasculature (Gale et al. 2002; Thomson et al. 2014), and in transgenic mice, where ANGPT2 is expressed by endothelial cells in noninflammatory conditions (Kim et al. 2016; Korhonen et al. 2016). However, loss of TIE1 in conditional TIE1 knockout mice or during inflammation switched ANGPT2 into a TIE2 antagonist, indicating that ANGPT2 activity can be modulated by several mechanisms (Kim et al. 2016; Korhonen et al. 2016).

The levels of VEGF are known to regulate ANGPT2-dependent cellular responses. During early growth of glioma tumor cells in rodent models, ANGPT2 was reported to destabilize glioma co-opted blood vessels, before upregulation of VEGF, resulting in a secondary avascular tumor. In this hypoxic environment VEGF expression was increased, and VEGF stimulated tumor angiogenesis in synergy with ANGPT2 (Holash et al. 1999). In an analogous manner, during ischemia-induced pathological neovascularization in retina, ANGPT2 induced vessel regression when VEGF levels were low, but enhanced neovascularization when VEGF levels 
were high (Oshima et al. 2005). Yet, ANGPT2 has been shown to act as an agonist in some tumor models. REGN910, a human antibody generated against the C-terminal FLD of ANGPT2, inhibits the interaction between ANGPT2 and TIE2 (Daly et al. 2013). REGN910 inhibited tumor growth in colorectal and epidermoid tumor xenograft models. Interestingly, ANGPT1 prevented the beneficial effect of REGN910 on tumor growth inhibition, suggesting that ANGPT2 served as a TIE2 agonist in these tumor models. Additionally, the expression of some TIE2 repressed genes was increased after REGN910 treatment but completely reversed with additional ANGPT1 treatment.

\section{Angiopoietins in Human Cancer}

ANGPT2 is expressed in conditions of vascular remodeling and indeed especially the circulating ANGPT2 has been found highly upregulated in a large variety of human tumors, specifically in advanced stages of cancer. In fact, the possibility to use ANGPT2 as marker for progressive tumors has been intensively investigated. Due to the challenges related to immunohistochemical staining of ANGPT2 in patients' samples, most of the evidence of ANGPT2 upregulation in cancer patients come from the increased soluble ANGPT2 levels in plasma or mRNA levels in tissue. It remains to be determined, whether the circulating ANGPT2 levels or the ANGPT2 mRNA expression correlates with the expression of ANGPT2 protein in endothelial cells of the tumor vasculature.

The increased circulating ANGPT2 levels have been observed in many human cancers and linked to poor prognosis. In addition to circulating ANGPT2 levels, the expression of ANGPT2 mRNA or protein has been reported in various cancers. Mainly, the ANGPT2 expression is described in tumor ECs; however, some reports also indicate the ANGPT2 expression in tumor cells. In situ hybridization methods have been used to show increased ANGPT2 expression in ECs. In neuroendocrine tumors, where circulating levels of ANGPT2 correlated with advanced, metastatic disease and could be used to identify patients with high risk of rapid disease progression, ANGPT2 expression was detected in the vascular endothelium (Detjen et al. 2010). In colorectal cancer using laser capture microdissection, the ANGPT2 expression was located in stromal cells but not in tumor cells (Goede et al. 2010). In gliomas, ANGPT2 mRNA expression was restricted to a subset of blood vessels (Stratmann et al. 1998). In hepatocellular carcinoma, ANGPT2 mRNA expression was associated specifically with hypervascular tumors (Tanaka et al. 1999). In human breast cancer samples $A N G P T 2$ was expressed by endothelial compartment but also by tumor cells (Sfiligoi et al. 2003). In prostate cancer ANGPT2 was highly upregulated in tumor epithelial cells particularly in high-grade tumors and correlated with increased microvessel density, metastases, and poor clinical outcome (Lind et al. 2005).

ANGPT2 expression has been shown in renal cell carcinoma (RCC) (Currie et al. 2002; Rautiola et al. 2016; Wang et al. 2014). ANGPT2 expression was found specifically in the tumor endothelium with weaker or no expression in tumor cells (Currie et al. 2002; Rautiola et al. 2016). Specific endothelial expression was found to correlate with vascular density (Rautiola et al. 2016). In advanced RCC ANGPT2 expression has been investigated in the context of first-line sunitinib therapy targeting multiple receptor tyrosine kinases, including VEGFRs. Interestingly, high endothelial ANGPT2 expression and CD31 expression were associated with a beneficial clinical response to sunitinib but not with patient survival. Another study showed that very high ANGPT2 expression was in general associated with better survival of RCC patients who received no anti-angiogenic therapy (Lampinen et al. 2016). In line with this, high ANGPT2 was associated with lower tumor grade and stage in this patient cohort. In another study, low circulating ANGPT2 levels in RCC patients were associated with better response to sunitinib treatment (Motzer et al. 2014). The examination whether the circulating levels of ANGPT2 correspond to the ANGPT2 expression levels in endothelial cells is needed to shed light on these results. In 
another study, plasma ANGPT2 levels were significantly higher in patients with mRCC compared to healthy individuals or patients with stage I disease (Wang et al. 2014). Patient plasma ANGPT2 levels were observed to decrease during sunitinib therapy. Interestingly, the plasma ANGPT2 levels increased upon development of sunitinib treatment resistance. The correlation between ANGPT2 expression and sunitinib treatment response was not investigated. Additionally, in the majority of patients, circulating ANGPT2 levels increased during disease progression. Evaluation of ANGPT2 as a biomarker in phase 3 trial in gastric cancer identified baseline plasma ANGPT2 as an independent prognostic factor for overall survival. ANGPT2 was also associated with the incidence of liver metastasis. However, ANGPT2 did not predict bevacizumab (VEGF blocking antibody) response, nor did ANGPT2 levels increase upon progression (Hacker et al. 2016). Altogether, these data call for better understanding of ANGPT2 expression in human cancer and the correlation of tumor expressed ANGPT2 with circulating ANGPT2 levels. Furthermore, other tumor microenvironment parameters, such as hypoxia and inflammation may modulate the outcome of ANGPT2 signaling and the response to VEGF signaling inhibitors.

In glioblastoma, bevacizumab therapy was associated with decreased tumor vessel density when compared to the treatment-naïve glioblastoma (Scholz et al. 2016). Circulating ANGPT2 levels did not change in patients before and after bevacizumab treatment and in fact, bevacizumabtreated tumor vasculature was observed to highly express ANGPT2. Bevacizumab treatment was also associated with a relative increase of proangiogenic M2 polarized macrophages (Scholz et al. 2016). Whether the increased ANGPT2 expression and recruitment of tumor infiltrating macrophages upon bevacizumab treatment in human glioblastoma, and possible induction of therapy resistance, are linked together as suggested by data obtained from xenograft experiments remains to be determined.

Preclinical evidence has led to the development of several pharmacological ways to block angiopoietin signaling, which have progressed in clinical trials. Despite the strong preclinical data, the clinical trials have not yet been able to live up with the expectations. For example, trebananib, the ANGPT1 and ANGPT2 inhibiting peptibody, failed to improve the overall survival in combination with paclitaxel in the first phase 3 study (TRINOVA-1) with patients with ovarian cancer, although the addition of trebananib extended the progression-free survival in the same study (Monk et al. 2014, 2016). In the second study (TRINOVA-2) trebananib together with pegylated liposomal doxorubicin failed to increase progression-free survival, although trebananib increased objective response rate and duration of response (Marth et al. 2017). Although the clinical benefit of angiopoietin inhibition in clinical trials is yet to be discovered, the trials have demonstrated tolerable toxicity profile, distinct from that of VEGF inhibitors. In addition, angiopoietin-targeted drugs are tested in human cancer in combination with VEGF targeted therapy, and similarly to the combination with chemotherapy, the combination with anti-angiogenic therapy has proven tolerable in phase 2 trials (Atkins et al. 2015). In addition, MEDI3617, an anti-ANGPT2 antibody, and vanucizumab, a bispecific ANGPT2 and VEGF blocking antibody, are currently being tested in combination with immune checkpoint inhibitors in human cancer (www.clinicaltrials.gov). The results will reveal a potentially interesting concept of blocking the pro-inflammatory ANGPT2 with immune therapy.

In addition to cancer, excess growth of leaky neovessels occurs in other diseases, including vision-impairing diseases of the eye such as wet age-related macular degeneration (wAMD). VEGF-targeted anti-angiogenic therapy is widely used to treat wAMD that can lead to vision loss unless treated. Although effective in a large group of patients, in approximately $40 \%$ of patients the disease can progress despite of VEGF-targeted therapy (Ferrara and Adamis 2016). Therefore, the vascular stabilizing and anti-angiogenic features of ANGPT-TIE targeted drugs are investigated in human wAMD and diabetic macular edema (an edema causing, vision impairing vascular complication that affects diabetic patients). 
These approaches include the VE-PTP inhibitor (AKB-9778), combinations of VEGF-targeted drugs with anti-ANGPT2 antibodies or bispecific antibodies targeting both ANGPT2 and VEGF.

\section{Summary}

Currently, angiopoietins are intensively studied for their properties of regulating vascular remodeling and vessel stability. Although ANGPT1 and ANGPT2 bind to the same TIE2 receptor, they tend to have opposing effects on mature blood vascular endothelium. While ANGPT1 acts as a classical agonist of TIE2 by activating the receptor, ANGPT2 acts as a context-dependent agonist or antagonist of TIE2. Therefore, ANGPT2 binding to TIE2 may occur without receptor activation and at the same time preventing ANGPT1 from activating TIE2. TIE2 activation results in suppression of EC inflammation and permeability, promoting EC survival and vessel stability. However, the angiopoietin signaling is complex, and it has been shown that EC secreted ANGPT2 has agonist activity, but this is lost during inflammation, leading to leaky vessels. In addition, the levels of VEGF have been reported to regulate ANGPT2 functions, low levels promoting vessel destabilization and high levels angiogenesis. Additionally, angiopoietins have been shown to signal via integrins, both in the presence and absence of TIE2, which may affect angiopoietin signaling in tumors, although this possibility remains to be further investigated. In addition, TIE1 was found critically required for ANGPT2 agonist activity. As TIE1 undergoes ectodomain cleavage in response to inflammatory stimuli, the level of TIE1 expression in tumors may also dictate ANGPT2 signaling outcomes. Understanding in which biological context, and possibly tumor types, ANGPT2 functions as an agonist or antagonist, or in which context it interacts with TIE2 or alternative integrin receptors, are key questions in order to harness angiopoietin signaling for therapeutic purposes.

ANGPT1 is necessary for correct vascular patterning and for the formation of nonleaky vessels of a functional vasculature. Whereas
ANGPT1 is important for proper investment of vessels with pericytes, ANGPT2 can destabilize vessels via inducing pericyte dropout, facilitating VEGF-dependent angiogenesis. Tumor vasculature is unorganized and leaky, which induces variation in the interstitial fluid pressure and consequently changes in blood flow, oxygenation, and drug distribution in the tumor. As a result, the tumor microenvironment is highly hypoxic, stimulating upregulation of hypoxiainducible genes VEGF, ANGPT2, and VE-PTP. In addition, VEGF further increases ANGPT2 expression. The leaky tumor vessels promote tumor cell dissemination and the abnormal tumor vasculature can fuel inflammation and limit antitumor immunity. ANGPT2 blocking has shown benefit in decreasing tumor metastasis, via several mechanisms and in combination with VEGF to modulate the inflammatory tumor microenvironment and to enhance anti-tumor immunity.

Although many studies have shown the beneficial effect of blocking ANGPT2 in mouse tumors that is further enhanced when provided in combination with VEGF blocking drugs or with immune checkpoint therapy, the function of ANGPT2 as a target, or prognostic, or predictive biomarker in human cancer remains to be investigated. The knowledge on the biological and molecular mechanisms of the ANGPT-TIE system should facilitate the clinical development of investigational ANGPT2-targeted drugs for the treatment of human cancer, in combination with VEGF-targeted anti-angiogenic or immune therapies.

\section{Cross-References}

\footnotetext{
- Anti-angiogenic Cancer Therapy: Development of Resistance

- Combination of Anti-angiogenics and Other Targeted Therapies

- Mechanisms of Anti-angiogenic Therapy

- Mechanisms of Tumor Angiogenesis

- Pathology of Tumor Angiogenesis

- The Role of the VEGF Signaling Pathway in Tumor Angiogenesis
} 
Acknowledgments Work in the laboratory of PS was funded by the Academy of Finland (grant numbers 271845, 310075 to PS; 308663 to EAK); the Jenny and Antti Wihuri Foundation; the Sigrid Juselius Foundation; the Cancer Society of Finland; and the University of Helsinki.

\section{References}

Atkins MB, Gravis G, Drosik K et al (2015) Trebananib (AMG 386) in combination with Sunitinib in patients with metastatic renal cell cancer: an open-label, multicenter, phase II study. J Clin Oncol 33(30):3431-3438

Babaei S, Teichert-Kuliszewska K, Zhang Q et al (2003) Angiogenic actions of angiopoietin-1 require endothelium-derived nitric oxide. Am J Pathol 162 (6):1927-1936

Barton WA, Tzvetkova-Robev D, Miranda EP et al (2006) Crystal structures of the Tie 2 receptor ectodomain and the angiopoietin-2-Tie2 complex. Nat Struct Mol Biol 13(6):524-532

Baumer S, Keller L, Holtmann A et al (2006) Vascular endothelial cell-specific phosphotyrosine phosphatase (VE-PTP) activity is required for blood vessel development. Blood 107(12):4754-4762

Bergers G, Hanahan D (2008) Modes of resistance to antiangiogenic therapy. Nat Rev Cancer 8(8):592-603

Brown JL, Cao ZA, Pinzon-Ortiz M et al (2010) A human monoclonal anti-ANG2 antibody leads to broad antitumor activity in combination with VEGF inhibitors and chemotherapy agents in preclinical models. Mol Cancer Ther 9(1):145-156

Carmeliet P, Ferreira V, Breier G et al (1996) Abnormal blood vessel development and lethality in embryos lacking a single VEGF allele. Nature 380 (6573):435-439

Cascone I, Napione L, Maniero F et al (2005) Stable interaction between $\{$ alpha\} 5 \{beta 1 integrin and tie 2 tyrosine kinase receptor regulates endothelial cell response to ang-1. J Cell Biol 170:993-1004

Castel P, Carmona FJ, Grego-Bessa J et al (2016) Somatic PIK3CA mutations as a driver of sporadic venous malformations. Sci Transl Med 8(332):332-342

Castillo SD, Tzouanacou E, Zaw-Thin M et al (2016) Somatic activating mutations in Pik 3 ca cause sporadic venous malformations in mice and humans. Sci Transl Med 8(332):332-343

Cho CH, Kammerer RA, Lee HJ et al (2004) COMP-Ang1: a designed angiopoietin-1 variant with nonleaky angiogenic activity. Proc Natl Acad Sci U S A 101 (15):5547-5552

Chu M, Li T, Shen B (2016) Angiopoietin receptor Tie2 is required for vein specification and maintenance via regulating COUP-TFII. Elife 5: e21032

Coffelt SB, Tal AO, Scholz A et al (2010) Angiopoietin-2 regulates gene expression in TIE2-expressing monocytes and augments their inherent proangiogenic functions. Cancer Res 70(13):5270-5280

Coxon A, Bready J, Min H et al (2010) Context-dependent role of angiopoietin-1 inhibition in the suppression of angiogenesis and tumor growth: implications for AMG 386 , an angiopoietin-1/2-neutralizing peptibody. Mol Cancer Ther 9(10):2641-2651

Currie MJ, Gunningham SP, Turner K et al (2002) Expression of the angiopoietins and their receptor Tie2 in human renal clear cell carcinomas; regulation by the von Hippel-Lindau gene and hypoxia. J Pathol 198 (4):502-510

D’Amico G, Korhonen EA, Anisimov A et al (2014) Tie1 deletion inhibits tumor growth and improves angiopoietin antagonist therapy. J Clin Invest 124 (2):824-834

Dalton AC, Shlamkovitch T, Papo N et al. (2016) Constitutive association of Tie1 and Tie2 with endothelial integrins is functionally modulated by angiopoietin-1 and fibronectin. PLoS One 11:e0163732

Daly C, Wong V, Burova E et al (2004) Angiopoietin-1 modulates endothelial cell function and gene expression via the transcription factor FKHR (FOXO1). Genes Dev 18(9):1060-1071

Daly C, Pasnikowski E, Burova E et al (2006) Angiopoietin-2 functions as an autocrine protective factor in stressed endothelial cells. Proc Natl Acad Sci U S A 103(42):15491-15496

Daly C, Eichten A, Castanaro C et al (2013) Angiopoietin2 functions as a Tie2 agonist in tumor models, where it limits the effects of VEGF inhibition. Cancer Res 73(1): 108-118

David S, Mukherjee A, Ghosh CC et al (2012) Angiopoietin-2 may contribute to multiple organ dysfunction and death in sepsis*. Crit Care Med 40(11): 3034-3041

Davis S, Aldrich TH, Jones PF et al (1996) Isolation of angiopoietin-1, a ligand for the TIE2 receptor, by secretion-trap expression cloning. Cell 87(7): 1161-1169

Davis S, Papadopoulos N, Aldrich TH et al (2003) Angiopoietins have distinct modular domains essential for receptor binding, dimerization and superclustering. Nat Struct Biol 10(1):38-44

De Palma M, Venneri MA, Galli R et al (2005) Tie2 identifies a hematopoietic lineage of proangiogenic monocytes required for tumor vessel formation and a mesenchymal population of pericyte progenitors. Cancer Cell 8(3):211-226

De Palma M, Biziato D, Petrova TV (2017) Microenvironmental regulation of tumour angiogenesis. Nat Rev Cancer 17:457-474

del Toro R, Prahst C, Mathivet T et al (2010) Identification and functional analysis of endothelial tip cell-enriched genes. Blood 116(19):4025-4033

Detjen KM, Rieke S, Deters A et al (2010) Angiopoietin-2 promotes disease progression of neuroendocrine tumors. Clin Cancer Res 16(2):420-429 
Dominguez MG, Hughes VC, Pan L et al (2007) Vascular endothelial tyrosine phosphatase (VE-PTP)-null mice undergo vasculogenesis but die embryonically because of defects in angiogenesis. Proc Natl Acad Sci U S A 104:3243-3248

Dumont DJ, Gradwohl G, Fong GH et al (1994) Dominantnegative and targeted null mutations in the endothelial receptor tyrosine kinase, tek, reveal a critical role in vasculogenesis of the embryo. Genes Dev 8(16): 1897-1909

Falcon BL, Hashizume H, Koumoutsakos P et al (2009) Contrasting actions of selective inhibitors of angiopoietin-1 and angiopoietin-2 on the normalization of tumor blood vessels. Am J Pathol 175(5):2159-2170

Felcht M, Luck R, Schering A et al (2012) Angiopoietin-2 differentially regulates angiogenesis through TIE2 and integrin signaling. J Clin Invest 122(6):1991-2005

Ferrara N, Adamis AP (2016) Ten years of anti-vascular endothelial growth factor therapy. Nat Rev Drug Discov 15:385

Ferrara N, Carver-Moore K, Chen H et al (1996) Heterozygous embryonic lethality induced by targeted inactivation of the VEGF gene. Nature 380(6573):439-442

Fiedler U, Scharpfenecker M, Koidl S et al (2004) The Tie-2 ligand angiopoietin-2 is stored in and rapidly released upon stimulation from endothelial cell Weibel-Palade bodies. Blood 103(11):4150-4156

Fiedler U, Reiss Y, Scharpfenecker M et al (2006) Angiopoietin-2 sensitizes endothelial cells to TNF-alpha and has a crucial role in the induction of inflammation. Nat Med 12(2):235-239

Frye M, Dierkes M, Kuppers V et al (2015) Interfering with VE-PTP stabilizes endothelial junctions in vivo via Tie-2 in the absence of VE-cadherin. J Exp Med 212 (13):2267-2287

Fukuhara S, Sako K, Minami T et al (2008) Differential function of Tie2 at cell-cell contacts and cellsubstratum contacts regulated by angiopoietin-1. Nat Cell Biol 10(5):513-526

Gale NW, Thurston G, Hackett SF et al (2002) Angiopoietin-2 is required for postnatal angiogenesis and lymphatic patterning, and only the latter role is rescued by Angiopoietin-1. Dev Cell 3(3):411-423

Ghosh CC, David S, Zhang R et al (2016) Gene control of tyrosine kinase TIE2 and vascular manifestations of infections. Proc Natl Acad Sci U S A 113 (9):2472-2477

Goede V, Coutelle O, Neuneier J et al (2010) Identification of serum angiopoietin-2 as a biomarker for clinical outcome of colorectal cancer patients treated with bevacizumab-containing therapy. $\mathrm{Br} \mathrm{J}$ Cancer 103 (9):1407-1414

Goel S, Gupta N, Walcott BP et al (2013) Effects of vascular-endothelial protein tyrosine phosphatase inhibition on breast cancer vasculature and metastatic progression. J Natl Cancer Inst 105(16):1188-1201

Hacker UT, Escalona-Espinosa L, Consalvo N et al. (2016) Evaluation of angiopoietin-2 as a biomarker in gastric cancer: results from the randomised phase III AVAGAST trial. Br J Cancer 114:855-862

Hakanpaa L, Sipila T, Leppanen VM et al (2015) Endothelial destabilization by angiopoietin-2 via integrin beta1 activation. Nat Commun 6:5962

Harney AS, Karagiannis GS, Pignatelli J et al. (2017) The selective Tie2 inhibitor rebastinib blocks recruitment and function of Tie2Hi macrophages in breast cancer and pancreatic neuroendocrine tumors. Mol Cancer Ther 16:2486-2501.

Hashizume H, Falcon BL, Kuroda T et al (2010) Complementary actions of inhibitors of angiopoietin-2 and VEGF on tumor angiogenesis and growth. Cancer Res 70(6):2213-2223

Hayashi M, Majumdar A, Li X et al (2013) VE-PTP regulates VEGFR2 activity in stalk cells to establish endothelial cell polarity and lumen formation. Nat Commun 4:1672

Herbst RS, Hong D, Chap L et al (2009) Safety, pharmacokinetics, and antitumor activity of AMG 386, a selective angiopoietin inhibitor, in adult patients with advanced solid tumors. J Clin Oncol 27(21):3557-3565

Holash J, Maisonpierre PC, Compton D et al (1999) Vessel cooption, regression, and growth in tumors mediated by angiopoietins and VEGF. Science 284 (5422):1994-1998

Holopainen T, Huang H, Chen C et al (2009) Angiopoietin1 overexpression modulates vascular endothelium to facilitate tumor cell dissemination and metastasis establishment. Cancer Res 69(11):4656-4664

Holopainen T, Saharinen P, D'Amico G et al (2012) Effects of angiopoietin-2-blocking antibody on endothelial cell-cell junctions and lung metastasis. J Natl Cancer Inst 104(6):461-475

Im JH, Tapmeier T, Balathasan L et al (2013) G-CSF rescues tumor growth and neo-angiogenesis during liver metastasis under host angiopoietin-2 deficiency. Int J Cancer 132(2):315-326

Imanishi Y, Hu B, Jarzynka MJ et al (2007) Angiopoietin-2 stimulates breast cancer metastasis through the alpha(5) beta(1) integrin-mediated pathway. Cancer Res 67 (9):4254-4263

Ito K, Turcotte R, Cui J et al (2016) Self-renewal of a purified Tie2+ hematopoietic stem cell population relies on mitochondrial clearance. Science 354 (6316):1156-1160

Ivaska J, Heino J (2011) Cooperation between integrins and growth factor receptors in signaling and endocytosis. Annu Rev Cell Dev Biol 27:291-320

Jeansson M, Gawlik A, Anderson G et al (2011) Angiopoietin-1 is essential in mouse vasculature during development and in response to injury. $\mathrm{J}$ Clin Invest 121(6):2278-2289

Jones N, Dumont DJ (1998) The Tek/Tie2 receptor signals through a novel Dok-related docking protein, Dok-R. Oncogene 17(9):1097-1108

Keskin D, Kim J, Cooke VG et al (2015) Targeting vascular pericytes in hypoxic tumors increases lung metastasis via angiopoietin-2. Cell Rep 10(7):1066-1081 
Kienast Y, Klein C, Scheuer W et al (2013) Ang-2-VEGFA CrossMab, a novel bispecific human IgG1 antibody blocking VEGF-A and Ang-2 functions simultaneously, mediates potent antitumor, antiangiogenic, and antimetastatic efficacy. Clin Cancer Res 19(24): 6730-6740

Kim I, Kwak HJ, Ahn JE et al (1999) Molecular cloning and characterization of a novel angiopoietin family protein, angiopoietin-3. FEBS Lett 443(3):353-356

Kim KT, Choi HH, Steinmetz MO et al (2005) Oligomerization and multimerization is critical for angiopoietin1 to bind and phosphorylate tie2. J Biol Chem 280:20126-20131

Kim OH, Kang GH, Noh H et al (2013) Proangiogenic TIE2(+)/CD31 (+) macrophages are the predominant population of tumor-associated macrophages infiltrating metastatic lymph nodes. Mol Cells 36(5):432-438

Kim M, Allen B, Korhonen EA et al (2016) Opposing actions of angiopoietin-2 on Tie2 signaling and FOXO1 activation. J Clin Invest 126(9):3511-3525

Kim J, Park DY, Bae H et al (2017) Impaired angiopoietin/ Tie2 signaling compromises Schlemm's canal integrity and induces glaucoma. J Clin Invest. 127 (10):3877-3896.

Kloepper J, Riedemann L, Amoozgar Z et al (2016) Ang-2/ VEGF bispecific antibody reprograms macrophages and resident microglia to anti-tumor phenotype and prolongs glioblastoma survival. Proc Natl Acad Sci U S A 113(16):4476-4481

Kontos CD, Stauffer TP, Yang WP et al (1998) Tyrosine 1101 of Tie 2 is the major site of association of p85 and is required for activation of phosphatidylinositol 3-kinase and Akt. Mol Cell Biol 18(7):4131-4140

Korhonen J, Partanen J, Armstrong E et al (1992) Enhanced expression of the tie receptor tyrosine kinase in endothelial cells during neovascularization. Blood 80(10):2548-2555

Korhonen EA, Lampinen A, Giri H et al (2016) Tie1 controls angiopoietin function in vascular remodeling and inflammation. J Clin Invest 126(9):3495-3510

Lampinen AM, Virman JP, Bono P et al (2016) Novel angiogenesis markers as long-term prognostic factors in patients with renal cell cancer. Clin Genitourin Cancer 15(1):e15-e24

Lanitis E, Irving M, Coukos G (2015) Targeting the tumor vasculature to enhance $\mathrm{T}$ cell activity. Curr Opin Immunol 33:55-63

Lee HJ, Cho CH, Hwang SJ et al (2004) Biological characterization of angiopoietin-3 and angiopoietin-4. FASEB J 18(11):1200-1208

Lee J, Kim KE, Choi DK et al (2013) Angiopoietin-1 guides directional angiogenesis through integrin alphavbeta5 signaling for recovery of ischemic retinopathy. Sci Transl Med 5(203):203-127

Lee HS, SJ O, Lee KH et al (2014) Gln-362 of angiopoietin-2 mediates migration of tumor and endothelial cells through association with alpha5beta1 integrin. J Biol Chem 289(45):31330-31340
Leppanen VM, Saharinen P, Alitalo K (2017) Structural basis of Tie2 activation and Tie2/Tie1 heterodimerization. Proc Natl Acad Sci U S A 114 (17):4376-4381

Limaye N, Wouters V, Uebelhoer M et al (2009) Somatic mutations in angiopoietin receptor gene TEK cause solitary and multiple sporadic venous malformations. Nat Genet 41(1):118-124

Lin P, Buxton JA, Acheson A et al (1998) Antiangiogenic gene therapy targeting the endothelium-specific receptor tyrosine kinase Tie2. Proc Natl Acad Sci U S A 95(15):8829-8834

Lind AJ, Wikstrom P, Granfors T et al (2005) Angiopoietin 2 expression is related to histological grade, vascular density, metastases, and outcome in prostate cancer. Prostate 62(4):394-399

Macdonald PR, Progias P, Ciani B et al (2006) Structure of the extracellular domain of Tie receptor tyrosine kinases and localization of the angiopoietin-binding epitope. J Biol Chem 281(38):28408-28414

Maisonpierre PC, Suri C, Jones PF et al (1997) Angiopoietin-2, a natural antagonist for Tie2 that disrupts in vivo angiogenesis. Science 277(5322):55-60

Master Z, Jones N, Tran J et al (2001) Dok-R plays a pivotal role in angiopoietin-1-dependent cell migration through recruitment and activation of Pak. EMBO J 20 (21):5919-5928

Marth C, Vergote I, Scambia G et al. (2017) ENGOT-ov-6/ TRINOVA-2: Randomised, double-blind, phase 3 study of pegylated liposomal doxorubicin plus trebananib or placebo in women with recurrent partially platinum-sensitive or resistant ovarian cancer. Eur $\mathrm{J}$ Cancer 70:111-121

Mazzieri R, Pucci F, Moi D et al (2011) Targeting the ANG2/TIE2 Axis inhibits tumor growth and metastasis by impairing angiogenesis and disabling rebounds of Proangiogenic myeloid cells. Cancer Cell 19(4): 512-526

Mellberg S, Dimberg A, Bahram F et al (2009) Transcriptional profiling reveals a critical role for tyrosine phosphatase VE-PTP in regulation of VEGFR2 activity and endothelial cell morphogenesis. FASEB J 23 (5):1490-1502

Michael IP, Orebrand M, Lima M et al. (2017) Angiopoietin-1 deficiency increases tumor metastasis in mice. BMC Cancer 17:539-550.

Minami T, Jiang S, Schadler K et al (2013) The calcineurin-NFAT-angiopoietin-2 signaling axis in lung endothelium is critical for the establishment of lung metastases. Cell Rep 4(4):709-723

Monk BJ, Poveda A, Vergote I et al (2014) Antiangiopoietin therapy with trebananib for recurrent ovarian cancer (TRINOVA-1): a randomised, multicentre, double-blind, placebo-controlled phase 3 trial. Lancet Oncol 15(8):799-808

Monk BJ, Poveda A, Vergote I et al (2016) Final results of a phase 3 study of trebananib plus weekly paclitaxel in recurrent ovarian cancer (TRINOVA-1): long-term 
survival, impact of ascites, and progression-free survival-2. Gynecol Oncol 143(1):27-34

Moore JO, Lemmon MA, Ferguson KM (2017) Dimerization of Tie 2 mediated by its membrane-proximal FNIII domains. Proc Natl Acad Sci U S A 114 (17):4382-4387

Motzer RJ, Hutson TE, Hudes GR et al (2014) Investigation of novel circulating proteins, germ line singlenucleotide polymorphisms, and molecular tumor markers as potential efficacy biomarkers of first-line sunitinib therapy for advanced renal cell carcinoma. Cancer Chemother Pharmacol 74(4):739-750

Murdoch C, Tazzyman S, Webster S et al (2007) Expression of Tie- 2 by human monocytes and their responses to angiopoietin-2. J Immunol 178(11):7405-7411

Nasarre P, Thomas M, Kruse K et al (2009) Host-derived angiopoietin-2 affects early stages of tumor development and vessel maturation but is dispensable for later stages of tumor growth. Cancer Res 69(4):1324-1333

Oh N, Kim K, Kim SJ et al (2015) A designed Angiopoietin-1 variant, dimeric CMP-Ang1 activates Tie2 and stimulates angiogenesis and vascular stabilization in N-glycan dependent manner. Sci Rep 5:15291

Oliner J, Min H, Leal J et al (2004) Suppression of angiogenesis and tumor growth by selective inhibition of angiopoietin-2. Cancer Cell 6(5):507-516

Oshima Y, Oshima S, Nambu H et al (2005) Different effects of angiopoietin-2 in different vascular beds: new vessels are most sensitive. FASEB J 19(8): 963-965

Park JS, Kim IK, Han S et al (2017) Normalization of tumor vessels by Tie2 activation and Ang2 inhibition enhances drug delivery and produces a favorable tumor microenvironment. Cancer Cell 31(1):157-158

Peterson TE, Kirkpatrick ND, Huang Y et al (2016) Dual inhibition of Ang-2 and VEGF receptors normalizes tumor vasculature and prolongs survival in glioblastoma by altering macrophages. Proc Natl Acad Sci U S A 113(16):4470-4475

Porat RM, Grunewald M, Globerman A et al (2004) Specific induction of tie1 promoter by disturbed flow in atherosclerosis-prone vascular niches and flowobstructing pathologies. Circ Res 94(3):394-401

Puri MC, Bernstein A (2003) Requirement for the TIE family of receptor tyrosine kinases in adult but not fetal hematopoiesis. Proc Natl Acad Sci U S A 100 (22):12753-12758

Puri MC, Rossant J, Alitalo K et al (1995) The receptor tyrosine kinase TIE is required for integrity and survival of vascular endothelial cells. EMBO J 14(23): 5884-5891

Puri MC, Partanen J, Rossant J et al (1999) Interaction of the TEK and TIE receptor tyrosine kinases during cardiovascular development. Development 126(20): 4569-4580

Qu X, Tompkins K, Batts LE et al (2010) Abnormal embryonic lymphatic vessel development in Tie1 hypomorphic mice. Development 137(8):1285-1295
Qu XH, Zhou B, Baldwin HS (2015) Tie1 is required for lymphatic valve and collecting vessel development. Dev Biol 399(1):117-128

Rasmussen AL, Okumura A, Ferris MT et al (2014) Host genetic diversity enables Ebola hemorrhagic fever pathogenesis and resistance. Science 346 (6212):987-991

Rautiola J, Lampinen A, Mirtti T et al (2016) Association of angiopoietin-2 and $\mathrm{Ki}-67$ expression with vascular density and sunitinib response in metastatic renal cell carcinoma. PLoS One 11(4):e0153745

Reiss Y, Droste J, Heil M et al (2007) Angiopoietin-2 impairs revascularization after limb ischemia. Circ Res 101(1):88-96

Reiss Y, Knedla A, Tal AO et al (2009) Switching of vascular phenotypes within a murine breast cancer model induced by angiopoietin-2. J Pathol 217(4):571-580

Rigamonti N, Kadioglu E, Keklikoglou I et al (2014) Role of angiopoietin-2 in adaptive tumor resistance to VEGF signaling blockade. Cell Rep 8(3):696-706

Saharinen P, Kerkela K, Ekman N et al (2005) Multiple angiopoietin recombinant proteins activate the Tie1 receptor tyrosine kinase and promote its interaction with Tie2. J Cell Biol 169(2):239-243

Saharinen P, Eklund L, Miettinen J et al (2008) Angiopoietins assemble distinct Tie2 signalling complexes in endothelial cell-cell and cell-matrix contacts. Nat Cell Biol 10(5):527-537

Saharinen P, Eklund L, Alitalo K (2017) Therapeutic targeting of the angiopoietin-TIE pathway. Nat Rev Drug Discov 16:635-661

Savant S, La Porta S, Budnik A et al (2015) The orphan receptor Tie1 controls angiogenesis and vascular remodeling by differentially regulating Tie 2 in tip and stalk cells. Cell Rep 12(11):1761-1773

Schmittnaegel M, Rigamonti N, Kadioglu E et al (2017) Dual angiopoietin-2 and VEGFA inhibition elicits antitumor immunity that is enhanced by PD-1 checkpoint blockade. Sci Transl Med 9(385):eaak9670

Scholz A, Harter PN, Cremer S et al (2016) Endothelial cell-derived angiopoietin-2 is a therapeutic target in treatment-naive and bevacizumab-resistant glioblastoma. EMBO Mol Med 8(1):39-57

Schulz P, Fischer C, Detjen KM et al (2011) Angiopoietin2 drives lymphatic metastasis of pancreatic cancer. FASEB J 25(10):3325-3335

Sfiligoi C, de Luca A, Cascone I et al (2003) Angiopoietin2 expression in breast cancer correlates with lymph node invasion and short survival. Int J Cancer 103(4): 466-474

Shen B, Shang Z, Wang B et al (2014) Genetic dissection of tie pathway in mouse lymphatic maturation and valve development. Arterioscler Thromb Vasc Biol 34(6): 1221-1230

Souma T, Tompson SW, Thomson BR et al (2016) Angiopoietin receptor TEK mutations underlie primary congenital glaucoma with variable expressivity. J Clin Invest 126(7):2575-2587 
Sridharan V, Margalit DN, Lynch SA et al (2016) Effects of definitive chemoradiation on circulating immunologic angiogenic cytokines in head and neck cancer patients. J Immunother Cancer 4:32

Srivastava K, Hu J, Korn C et al (2014) Postsurgical adjuvant tumor therapy by combining antiangiopoietin-2 and metronomic chemotherapy limits metastatic growth. Cancer Cell 26(6):880-895

Stratmann A, Risau W, Plate KH (1998) Cell type-specific expression of angiopoietin-1 and angiopoietin-2 suggests a role in glioblastoma angiogenesis. Am J Pathol 153(5):1459-1466

Suri C, Jones PF, Patan S et al (1996) Requisite role of angiopoietin-1, a ligand for the TIE2 receptor, during embryonic angiogenesis. Cell 87(7):1171-1180

Tanaka S, Mori M, Sakamoto Y et al (1999) Biologic significance of angiopoietin-2 expression in human hepatocellular carcinoma. J Clin Invest 103 (3):341-345

Thomas M, Felcht M, Kruse K et al (2010) Angiopoietin-2 stimulation of endothelial cells induces alphavbeta3 integrin internalization and degradation. J Biol Chem 285(31):23842-23849

Thomas M, Kienast Y, Scheuer W et al (2013) A novel angiopoietin-2 selective fully human antibody with potent anti-tumoral and anti-angiogenic efficacy and superior side effect profile compared to Pan-Angiopoietin-1/-2 inhibitors. PLoS One 8(2): e54923

Thomson BR, Heinen S, Jeansson M et al (2014) A lymphatic defect causes ocular hypertension and glaucoma in mice. J Clin Invest 124(10):4320-4324

Thomson BR, Souma T, Tompson SW et al (2017) Angiopoietin-1 is required for Schlemm's canal development in mice and humans. J Clin Invest [Epub ahead of print]

Venneri MA, De Palma M, Ponzoni M et al (2007) Identification of proangiogenic TIE2-expressing monocytes (TEMs) in human peripheral blood and cancer. Blood 109(12):5276-5285

Wang X, Bullock AJ, Zhang L et al (2014) The role of angiopoietins as potential therapeutic targets in renal cell carcinoma. Transl Oncol 7(2):188-195

Welford AF, Biziato D, Coffelt SB et al (2011) TIE2expressing macrophages limit the therapeutic efficacy of the vascular-disrupting agent combretastatin A4 phosphate in mice. J Clin Invest 121(5):1969-1973

Wilhelm K, Happel K, Eelen G et al (2016) FOXO1 couples metabolic activity and growth state in the vascular endothelium. Nature 529(7585):216-220

Winderlich M, Keller L, Cagna G et al (2009) VE-PTP controls blood vessel development by balancing Tie-2 activity. J Cell Biol 185(4):657-671

Winkler F, Kozin SV, Tong RT et al (2004) Kinetics of vascular normalization by VEGFR2 blockade governs brain tumor response to radiation: role of oxygenation, angiopoietin-1, and matrix metalloproteinases. Cancer Cell 6(6):553-563

Woo KV, Qu X, Babaev VR et al (2011) Tiel attenuation reduces murine atherosclerosis in a dose-dependent and shear stress-specific manner. J Clin Invest 121 (4): $1624-1635$

Yuan HT, Khankin EV, Karumanchi SA et al (2009) Angiopoietin 2 is a partial agonist/antagonist of Tie2 signaling in the endothelium. Mol Cell Biol 29(8):2011-2022

Ziegler T, Horstkotte J, Schwab C et al (2013) Angiopoietin 2 mediates microvascular and hemodynamic alterations in sepsis. J Clin Invest 123:3436 